\title{
Proper Generalized Decomposition for Parameterized Helmholtz Problems in Heterogeneous and Unbounded Domains: Application to Harbor Agitation
}

D. Modesto S. Zlotnik A. Huerta 


\section{Proper Generalized Decomposition for Parameterized Helmholtz Problems in Heterogeneous and Unbounded Domains: Application to Harbor Agitation}
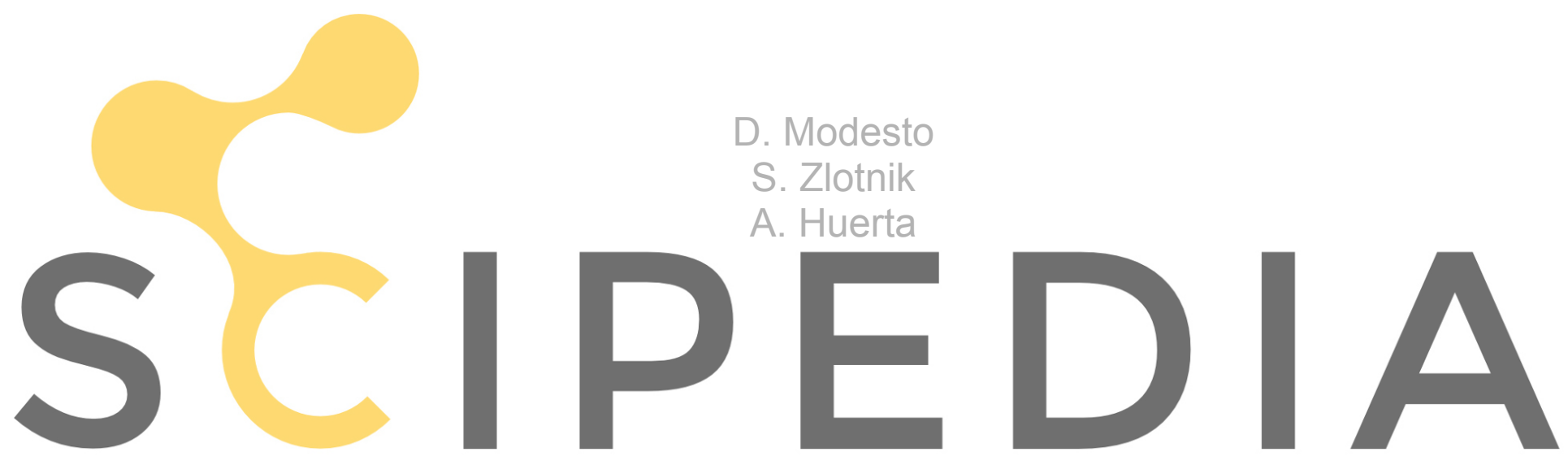

Register for free at https//www.scipedia.com to download the version without the watermark Publication CIMNE №-401, October 2013

International Center for Numerical Methods in Engineering Gran Capitán s/n, 08034 Barcelona, Spain 


\section{Proper generalized decomposition for parameterized Helmholtz problems in heterogeneous and unbounded domains: application to harbor agitation}

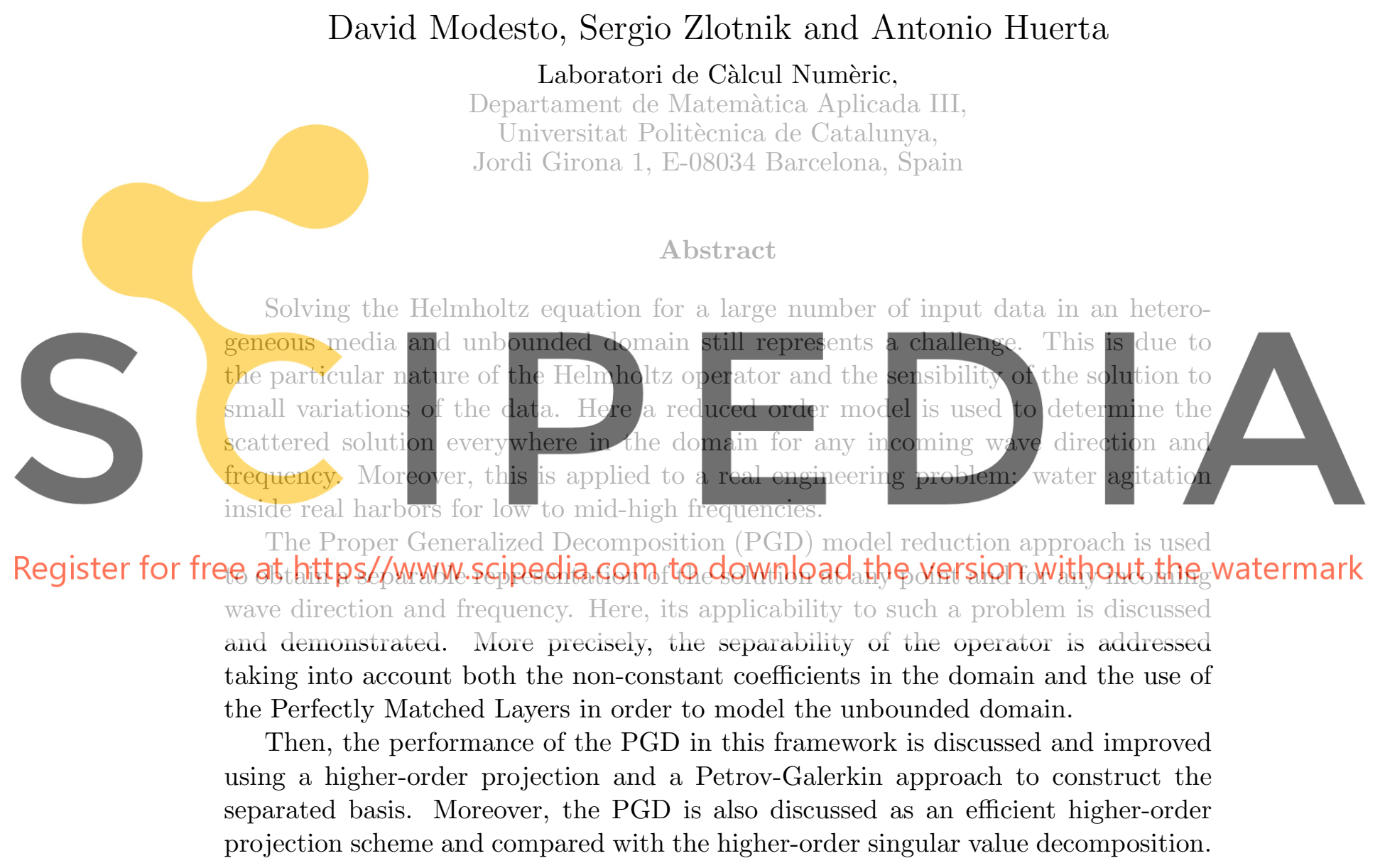

Keywords: Reduced Order Models, Proper Generalized Decomposition, Helmholtz, Parameterized solutions, wave propagation, harbor

\section{Introduction}

A large number of models involving the propagation of harmonic waves in unbounded domains are governed by Helmholtz-type partial differential equations. Their numerical 
solution is usually computationally demanding. Well-known difficulties are: pollution errors [33, 34, 24], treatment of the unbounded domain [49], and modeling small geometric features that have a large influence on the scattered field [45, 46]. Moreover, in engineering practice, wave propagation computations are usually one of many steps in a design process, an optimization strategy or an identification analysis. In summary, accuracy is compromised because the large computer costs drastically reduce the number (or range) of parameters tested. Note that the obvious approach of directly interpolating a few (costly) computed solutions to estimate results for intermediate parameter values is not viable because the solution is extremely sensitive to the parameters (e.g. incoming wave frequency and direction, geometry, etc.).

This paper proposes a strategy to reduce the computational limit imposed on the number of Helmholtz solutions that are feasible to compute in a design or optimization process. More precisely, the objective is to construct the generalized (high-dimensional) solution of a parameterized scattering problem in an heterogeneous and unbounded domain. This generalized solution, recently called computational vademecum [19] in a more general framework, provides the engineer a way to evaluate in real-time any tentative scattering situation (e.g. the Helmholtz solution and its derivatives). Therefore it extremely accelerates the process of evaluating solutions of the Helmholtz problem.

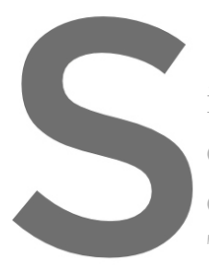

There are severa

is on the parameters

direction. Each of th

dependent) and is cons

This results in a hich
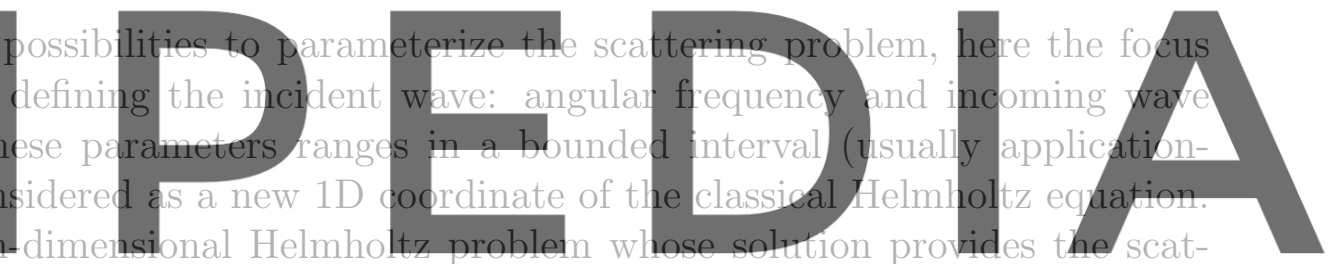

tered field at any point of the domain and for any incident condition. Moreover, the

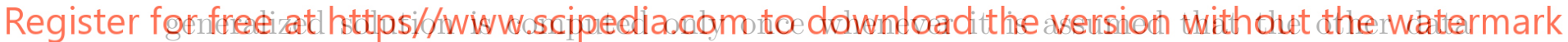

(geometry, boundary conditions, etc.) do not change, which is usual in most of engineering applications. The important point is that any subsequent evaluation of the scattered field is readily obtained by means of a fast post-process (this, for instance, can be the case of a time signal including a wide range of frequencies).

The high-dimensional character of the proposed problem involves an exponential growth of degrees of freedom (the so-called curse of dimensionality) when using standard meshbased discretization techniques. A reduced order model can circumvent this critical difficulty.

Here, the proper generalized decomposition (PGD) $[2,3]$ is used. This method has been studied and successfully applied to various problems in computational mechanics, see $[16,17,31,4,37]$. The interested reader is addressed to [42, 43, 18] and the references therein for a survey on different PGD techniques. PGD computes iteratively each term of the approximation using products of separable functions and reducing the highdimensional complexity of the original problem. Therefore, it is able to circumvent the curse of dimensionality and provide an approximation of the generalized solution. In contrast to classical a posteriori approaches, like POD [35, 38, 12] or reduced basis methods 
$[41,15,27]$, the PGD solution is evaluated online using a (fast) linear combination of the separated functions. In the present framework these functions are particularized for intermediate frequencies and incoming wave directions. This also contrasts with previous works in this field; see for instance [50], where an a posteriori reduced model for the homogeneous Helmholtz equation has to be constructed for each intermediate value. Moreover, the PGD does not require neither to precompute any trial solution (i.e. a snapshot) nor to solve a singular value decomposition. Note that this last point can even preclude the application of POD-based techniques for the Helmholtz problem if dense spatial discretizations are required.

The contributions in this paper are applicable to any heterogeneous and unbounded problem governed by the Helmholtz equation, and requiring a large number of evaluations of the input data. However the presented work is inspired on an engineering application: the prediction of water agitation inside harbors. Particularly, two harbors located in the Northeast of Spain are used as test cases. Note that the parameterized wave propagation problem becomes in this case 4D: two spatial coordinates, one for frequency and one for the incoming wave direction. Separated representations including the frequency as a dimension are not completely new, see for example [44]. However, in this case real-time evaluation of the PGD solution makes the proposed methodology an exceptional tool for harbor design

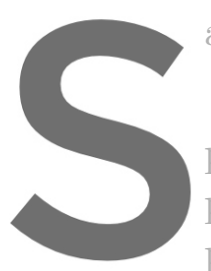

and study.

Some important points

problem require speci

putational domain nee

perfectly matched layers ( $\mathrm{P}$

a PGD framework. Second, the wave problem induces non-separable terms in the equa-

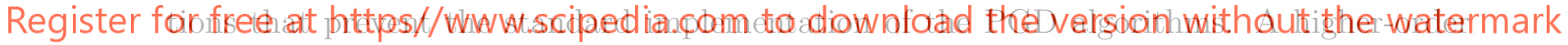

projection based on the PGD solution of a multidimensional equation is used to construct

an optimal separable wave problem. And finally, the non-hermitian character of the involved operator and the oscillatory nature of the wave field induce serious degradations in the convergence of the standard PGD techniques. Here, an improvement is proposed based on a Petrov-Galerkin approach, originally developed in [43], and on the use of the PGD-projection commented before.

\section{Problem statement}

The Helmholtz equation in an heterogeneous media and unbounded domain is considered. The application to harbors assumed here imposes the use of the mild slope equation (MSE) [11], which describes the motion of sea waves over a slow varying bottom depth, and allows to model the refraction and diffraction for deep and shallow waters. The MSE emanate from the incompressible Navier-Stokes equations (with the hypothesis of non-viscous fluid, small amplitude monochromatic waves and slow varying bottom), and is a common and 
useful tool for evaluating wave agitation in coastal zone and in harbors. In frequency domain they are written as

$$
\boldsymbol{\nabla} \cdot\left(c c_{g} \nabla u\right)+k^{2} c c_{g} u=0
$$

over an unbounded $2 \mathrm{D}$ domain where $u \in \mathbb{C}$ is the wave surface elevation, $k(h, \omega) \in \mathbb{R}$ is the wavenumber, $h(x, y) \in \mathbb{R}$ is the bathymetry (i.e. mean-water-level-depth), $\omega \in I_{\omega} \subset \mathbb{R}$ is the angular frequency of the monochromatic incoming wave, $c=\omega / k$ is the phase velocity and $c_{g}=g\left[\tanh (k h)+k h \operatorname{sech}^{2}(k h)\right] /(2 \omega)$ is the group velocity, where $g$ is the acceleration of gravity. It is important to note that the wavenumber, $k$, depends on bathymetry, $h$, and the frequency, $\omega$, by the so-called nonlinear dispersion relation

$$
\omega^{2}=k g \tanh (k h)
$$

which models the effect of the bathymetry on the wave propagation, that is, the refraction.

Boundary conditions are, on one hand, for reflecting/absorbing boundaries $\Gamma_{\mathrm{R}}$,

$$
\boldsymbol{n} \cdot c c_{g} \nabla u-i k c c_{g} \alpha u=0 \text { on } \Gamma_{\mathrm{R}},
$$

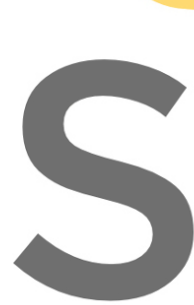

where $i=\sqrt{-1}$ is the

experimental coefficient

This coefficient is equ

absorbing boundaric

so-called Sommerfeld
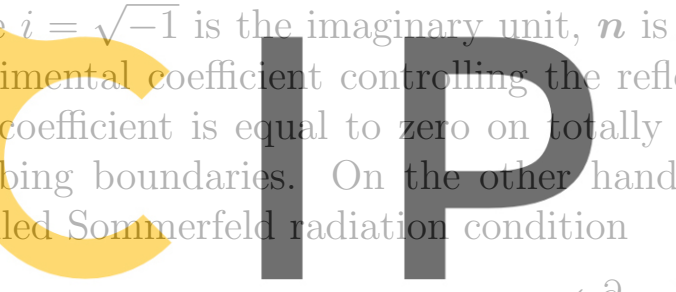

$\lim \sqrt{r}\left(\frac{\partial}{\partial r}-i k\right)\left(u-u_{0}\right)=0$,

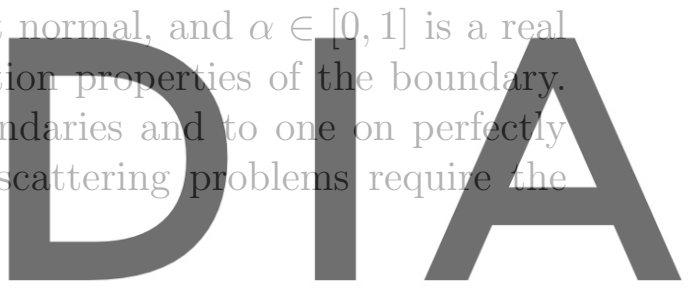

(4)

Register for free at https//www.scipedia.com to download the version without the watermark

where $r$ is the radial direction and $u_{0}$ the incident wave. This wave is defined on a constant far-field bathymetry $h_{0}$ by

$$
u_{0}=\exp \left(i k_{0} x \cos \theta\right) \exp \left(i k_{0} y \sin \theta\right) \in \mathbb{C},
$$

where $\theta \in I_{\theta} \subset \mathbb{R}$ is the imposed incoming wave direction (data) and $k_{0}$ is determined from dispersion relation (2) for $h=h_{0}$.

Remark 1 (amplitude of incoming wave). It is important to note that the amplitude of the incoming wave is unitary because the solution can be scaled thanks to the linearity of the problem. In fact, the amplitude of the solution $u$ is called the amplification factor because it should be scaled with the actual amplitude of the incoming wave to obtain the actual surface elevation.

The Sommerfeld radiation condition requires, in practice, the introduction of an artificial boundary and its corresponding boundary condition. Many methods have been proposed in the literature to deal with this situation. Among others, they include infinite elements [23], local non-reflecting boundary conditions (NRBC) [30], Dirichlet to Neumann 


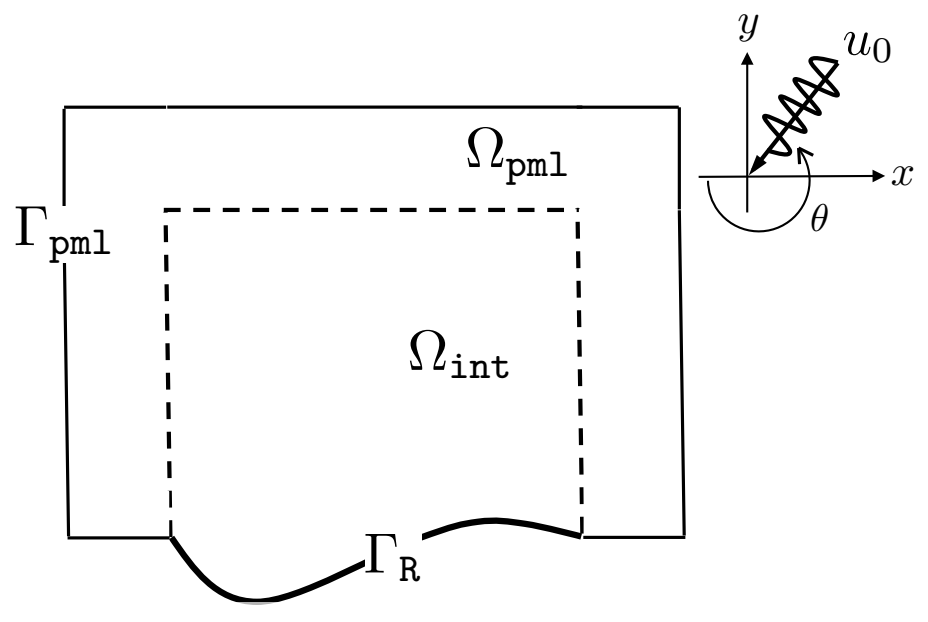

Figure 1: Computational domain sketch.

non-local operators [26, 29] and perfectly matched layers (PML) [9]. Here PML is chosen because of its exceptional properties, see for instance [40, 8, 47, 22], and its straightforward extension to multidimensional models. Moreover, a first order NRBC is placed on

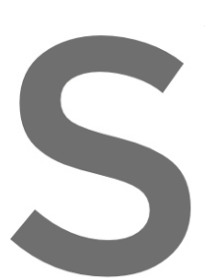
the artificial bound

\section{In this case it is} union of the region introduce an artificial $k$ are assumed constan
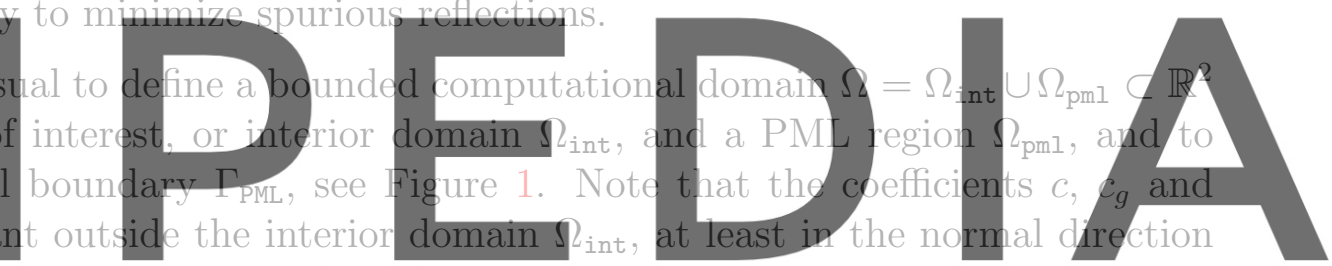

to $\Gamma_{\text {PML }}$. The Perfectly Matched Layer (PML) surrounds $\Omega_{\text {int }}$ in order to absorb outgoing

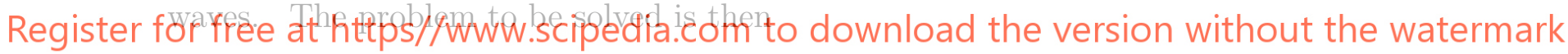

$$
\begin{aligned}
\nabla \cdot\left(c c_{g} \mathbb{P} \nabla u\right)+k^{2} c c_{g} s_{x} s_{y} u & =s(x, y, \omega, \theta) & & \text { in } \Omega, \\
\boldsymbol{n} \cdot\left(c c_{g} \mathbf{P} \nabla u\right)-i k c c_{g} \alpha u & =0 & & \text { on } \Gamma_{\mathrm{R}}, \\
\boldsymbol{n} \cdot\left(c c_{g} \mathbf{P} \nabla u\right)-i k c c_{g} u & =\boldsymbol{n} \cdot\left(c c_{g} \mathbf{P} \nabla u_{0}\right)-i k c c_{g} u_{0} & & \text { on } \Gamma_{\mathrm{PML}},
\end{aligned}
$$

where the non-homogenous term in (6a) is defined as

$$
s= \begin{cases}0 & \text { if }(x, y) \in \Omega_{\mathrm{int}}, \\ \boldsymbol{\nabla} \cdot\left(c c_{g} \mathbf{P} \boldsymbol{\nabla} u_{0}\right)+k^{2} c c_{g} s_{x} s_{y} u_{0} & \text { if }(x, y) \in \Omega_{\mathrm{pml}},\end{cases}
$$

to account for the incident wave and absorb only the scattered waves in the PML region. Equation (6c) is a first order non-reflecting boundary condition discretizing (4) on $\Gamma_{\mathrm{PML}}$, to minimize non-physical reflection from the PML outer boundary. Thus, $\partial \Omega=\bar{\Gamma}_{\mathrm{R}} \cup \bar{\Gamma}_{\mathrm{PML}}$ with $\Gamma_{\mathrm{R}} \cap \Gamma_{\mathrm{PML}}=\emptyset$, and no Dirichlet boundary conditions are imposed. The diagonal anisotropy matrix defining the absorption in the PML medium is denoted $\mathbf{P}$ and defined as

$$
\mathbf{P}=\left(\begin{array}{cc}
s_{y} / s_{x} & 0 \\
0 & s_{x} / s_{y}
\end{array}\right)
$$


where $s_{x}=1+\sigma_{x} / \omega$ and $s_{y}=1+\sigma_{y} / \omega$ are the absorption parameters in the two Cartesian directions. Note that $s_{x}=s_{y}=1$ outside the PML region because the absorbing functions $\sigma_{x}(x) \geq 0$ and $\sigma_{y}(y) \geq 0$ are zero in $\Omega_{\text {int }}$ and monotonic polynomials along the corresponding cartesian absorbing direction. More details on the application of the PML to the Helmholtz equation can be found in [47, 13] among others.

Remark 2 (separability of coefficients). It is important to note that the coefficients in (6) are non-constant. In general, they depend on the bathymetry, $h$ (and consequently on the spatial coordinates $x$ and $y$ ), the angular frequency of the monochromatic incoming wave, $\omega$, and the incoming wave direction, $\theta$. This dependence is, in general, nonlinear. But the crucial issue for the proposed methodology is that these coefficients are not expressed a priori as separable functions of the data: $h(x, y), \omega$, and $\theta$. They are not written a sum of products of functions of $h, \omega$, and $\theta$. Section 5 discusses this issue in more detail.

\section{The parameterized wave propagation weak form}

For a given geometry and bathymetry, $h(x, y)$, engineers are confronted with multiple evaluations of problem (6) for different values of the angular frequency and direction of the incoming wave, namel solution of (6) for any and fast queries. Befor four-dimensional problem created by considerif

The total surface elevation, $u(x, y, \omega, \theta)$, is now seen as a function of the spatial coordiRegister for free at hittos wave in their respective range of interest, namely $I_{\omega}$ and $I_{\theta}$. The variational problem equivalent to (6) requires finding $u$ for all $\delta u$ in the selected appropriate functional space such that

$$
A(u, \delta u)=L(\delta u)
$$

The non-hermitian bilinear form $A(\cdot, \cdot)$ and the linear form $L(\cdot)$ are defined by

$$
A(u, \delta u)=\int_{I_{\theta}} \int_{I_{\omega}} a(u, \delta u ; \omega) d \omega d \theta \quad \text { and } \quad L(\delta u)=\int_{I_{\theta}} \int_{I_{\omega}} \ell(\delta u ; \omega, \theta) d \omega d \theta,
$$

with $a(\cdot, \cdot ; \omega)$ bilinear and continuous form and $\ell(\cdot ; \omega, \theta)$ linear bounded functional for all parameters $(\omega, \theta) \in I_{\omega} \times I_{\theta}$. They are the classical Helmholtz spatial weak forms with the parameter dependence explicitly indicated, that is

$$
\begin{aligned}
a(u, \delta u ; \omega)=\left(k^{2} c c_{g} s_{x} s_{y} u, \delta u\right)_{\Omega}-\left(c c_{g} \mathbf{P} \nabla\right. & u, \nabla \delta u)_{\Omega} \\
& +i \alpha\left\langle k c c_{g} u, \delta u\right\rangle_{\Gamma_{\mathrm{R}}}+i\left\langle k c c_{g} u, \delta u\right\rangle_{\Gamma_{\mathrm{pm}} \mathrm{l}},
\end{aligned}
$$

and

$$
\ell(\delta u ; \omega, \theta)=(s, \delta u)_{\Omega}+\left\langle\boldsymbol{n} \cdot\left(c c_{g} \mathbf{P} \nabla u_{0}\right)-i k c c_{g} u_{0}, \delta u\right\rangle_{\Gamma_{\mathrm{pm} 1}} .
$$


In the previous and the following equations, $(\cdot, \cdot)_{D}$ denotes the $\mathcal{L}^{2}$ scalar product (for complex functions) in any domain $D$, while $\langle\cdot, \cdot\rangle_{B}$ also denotes the $\mathcal{L}^{2}$ scalar product of the traces over $B$.

\section{The proper generalized decomposition method (PGD)}

Helmholtz problems require fine discretizations of the spatial domain. Moreover, for loworder approximations dense meshes must be enhanced with stabilized formulations to control dispersion errors $[7,48,39]$. In spite of the improved efficiency of high-order approximations $[28,10]$ a large number of degrees of freedom (DOF) is nonetheless required. For instance, as shown in [28], $10^{5}$ DOF are necessary to attain one significant digit of accuracy with fifth-order finite elements in this MSE problem. Hence, applying a standard discretization technique to solve the 4D problem may easily require here $10^{9}$ DOF when 100 nodes are used for parameters $\theta$ and $\omega$. However, PGD may effectively approximate the solution of problem (9) with a 2D computational cost.

PGD imposes an approximation of the wave field $u(x, y, \omega, \theta)$ in a rank- $n$ separated representation, namely
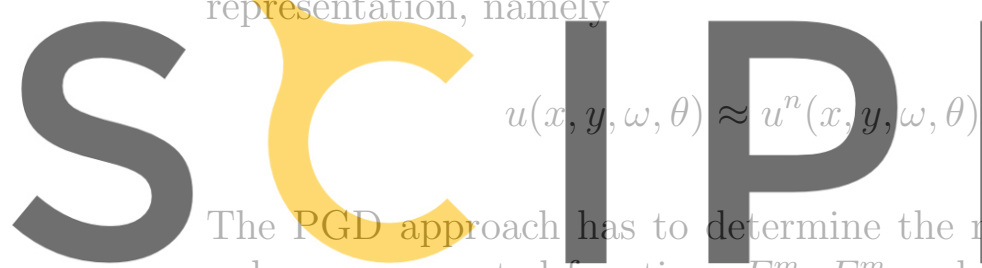

unknown separated functions $F_{1}^{m}, F_{2}^{m}$ and $F_{3}^{m}$ for $m=1$
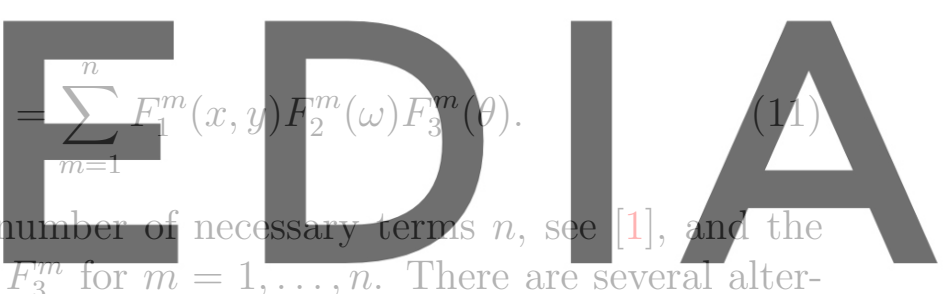

Register for free at https//Www.scipedia.com to download the version without the watermark $u^{n}(x, y, \omega, \theta)=u^{n-1}(x, y, \omega, \theta)+F_{1}(x, y) F_{2}(\omega) F_{3}(\theta)$,

where $u^{n-1}$ is assumed to be known, and $F_{1}, F_{2}$ and $F_{3}$ denote the separated functions of the unknown term (the superscript is dropped for the last term in order to alleviate notation). Replacing (12) in (9a) the following problem must be solved:

$$
A\left(F_{1} F_{2} F_{3}, \delta u\right)=L(\delta u)-A\left(u^{n-1}, \delta u\right) .
$$

Note that this represents a nonlinear problem for the unknowns $F_{1}, F_{2}$ and $F_{3}$.

A number of PGD approaches based on linearization techniques have been developed to solve the Eq. (13) efficiently, see for instance [43, 18]. Two of these approaches, which will be compared in Section 6, are detailed next.

\subsection{Standard PGD}

Consider the test functions $\delta u$ in Eq. (13) to be separated as

$$
\delta u=\delta F_{1} F_{2} F_{3}+F_{1} \delta F_{2} F_{3}+F_{1} F_{2} \delta F_{3} .
$$


This expression allows to approximate the solution with an alternating direction strategy (fixed point iteration). Each iteration requires to perform as many sequential steps as separated functions are used to approximate $u$. Here, the following three stages are iterated until convergence or a proxy for termination is reached (further commented in Section 6):

1. Assume that $F_{2}$ and $F_{3}$ are known $\left(\delta F_{2}=\delta F_{3}=0\right)$. Compute the linear problem to determine $F_{1} \in \mathcal{H}^{1}(\Omega)$, for all $\delta F_{1} \in \mathcal{H}^{1}(\Omega)$ satisfying

$$
A\left(F_{1} F_{2} F_{3}, \delta F_{1} F_{2} F_{3}\right)=L\left(\delta F_{1} F_{2} F_{3}\right)-A\left(u^{n-1}, \delta F_{1} F_{2} F_{3}\right) .
$$

Note that this step has a $2 \mathrm{D}$ cost. After solving (15a) the function $F_{1}$ is $\mathcal{L}^{2}$ normalized.

2. Assume now that $F_{1}$ and $F_{3}$ are known $\left(\delta F_{1}=\delta F_{3}=0\right)$. In fact, the solution of the previous step is used here in a Gauss-Seidel strategy. Solve a linear 1D problem to evaluate $F_{2} \in \mathcal{L}^{2}\left(I_{\omega}\right)$, for all $\delta F_{2} \in \mathcal{L}^{2}\left(I_{\omega}\right)$ satisfying

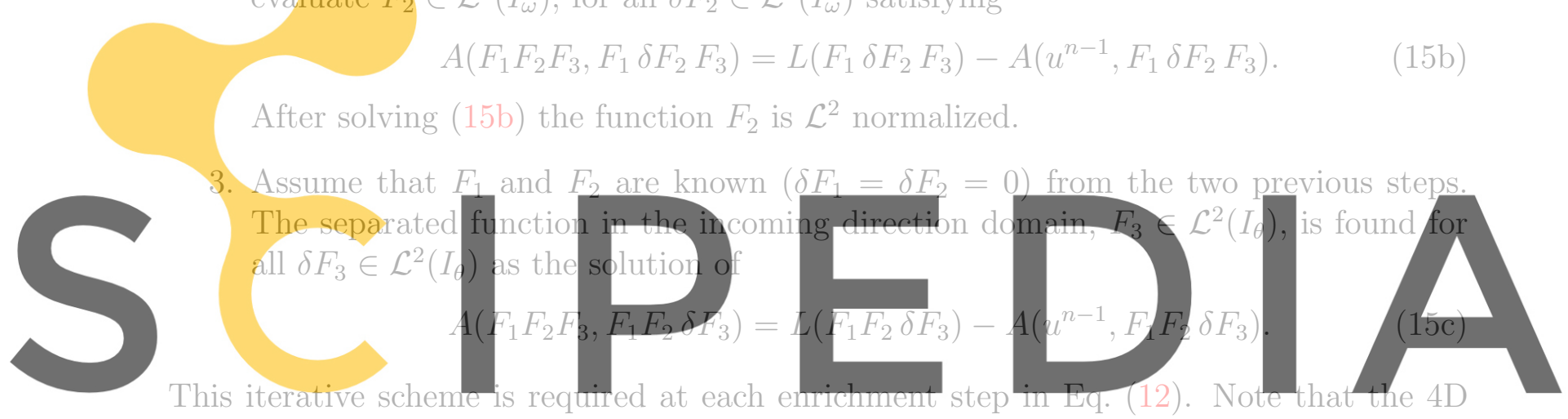

nature of the original problem is reduced to the iteration of one 2D problem (15a) and

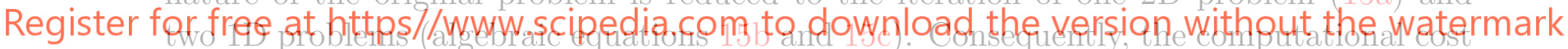

associated to the PGD approximation is the product of three factors: (i) the cost of the

2D Helmholtz solver, (ii) the total number of iterations performed and (iii) the number of terms involved in the separable representation of $u$. For the majority of elliptic problems, both, the number of iterations and the required terms are sufficiently small to ensure computational savings of several orders of magnitude, see for instance [3].

Unfortunately, the optimality of the standard approach is critically degraded for nonsymmetric operators, see [43]. This issue also applies to the non-hermitian MSE problem. Moreover, its complexity is increased due to the oscillatory nature of the solution. As shown in Section 6 the standard approach is, in general, not converging. The offline PGD constructor is imposed by a non-standard rationale introduced next.

Remark 3 (Algebraic equations). Note that steps 2 and 3, associated respectively to $\omega$ and $\theta$, can be solved pointwise because they are algebraic equations. That is, no derivatives with respect to $\omega$ or $\theta$ exist in the strong form of the problem, see (6). Since the choice of these sampling points is not trivial, here an approximation spaces for $F_{2}(\omega)$ and $F_{3}(\theta)$ are a priori defined for the weak form strategy in order to retain, when possible, a best approximation strategy over the whole range of the parameters. 


\subsection{Petrov-Galerkin PGD}

The Petrov-Galerkin PGD (PG PGD) is proposed in [43] and is applied here to recover the convergence of the PGD solution. Let us substitute the test functions $\delta u$ in Eq. (13) by $\delta \tilde{u}$, that is

$$
A\left(F_{1} F_{2} F_{3}, \delta \tilde{u}\right)=L(\delta \tilde{u})-A\left(u^{n-1}, \delta \tilde{u}\right)
$$

where $\delta \tilde{u}$ is defined by

$$
\delta \tilde{u}=\delta \widetilde{F}_{1} \widetilde{F}_{2} \widetilde{F}_{3}+\widetilde{F}_{1} \delta \widetilde{F}_{2} \widetilde{F}_{3}+\widetilde{F}_{1} \widetilde{F}_{2} \delta \widetilde{F}_{3}
$$

Functions $\widetilde{F}_{1}(x, y), \widetilde{F}_{2}(\omega)$ and $\widetilde{F}_{3}(\theta)$ are obtained by solving the auxiliary problem

$$
A\left(\delta u, \widetilde{F}_{1} \widetilde{F}_{2} \widetilde{F}_{3}\right)=\left(\delta u, F_{1} F_{2} F_{3}\right)_{\Omega \times I_{\omega} \times I_{\theta}},
$$

for all $\delta u$ in the form of Eq. (14).

Equations (16) and (17) are separable and therefore can be approximated using the same fixed point algorithm described before. Similarly to the standard PGD algorithm (15), the PG PGD approach requires solving a three stage procedure. In this case, however, each stage involves the solution of two equations: one to evaluate $\widetilde{F}_{i}$ and one to evaluate $F_{i}$, $i=1,2,3$. Note that each pair of equations induce the same matrix and therefore the computational cost is not duplicated.

After convergence of the fixed point algorithm, the auxiliary functions $\widetilde{F}_{1}, \widetilde{F}_{2}$ and $\widetilde{F}_{3}$ are not longer necessary. This algorithm was originally developed for non-symmetric convective-dominated problems with space-time decompositions, see [43]. It is known as a Petrov-Galerkin PGD because the improvement is induced by means of a modification of the test functions involved in the standard approach. Note that the implementation of PG PGD is a natural choice here since the MSE problem is non-hermitian. Numerical examples of Section 6 reveal that this approach is able to provide solutions in those cases where the standard one fails.

Remark 4 (convergence of PGD algorithms). For pure diffusive elliptic problems, the number of iterations in the fixed point algorithm typically does not exceed ten before the convergence criterion is fulfilled [2]. For more complex frameworks like the one proposed here, the new term $F_{1} F_{2} F_{3}$ may not converge and a maximum number of iterations needs to be imposed. It has been observed that if the maximum number of iterations is reduced, for instance to three, the PGD approximation may require more terms (more "modes") but the overall computational cost is drastically reduced.

\section{Separability of the MSE}

PGD requires the operators $A(\cdot, \cdot)$ and $L(\cdot)$, see Eqs. (9) and (10), to be expressed in a separable form. Otherwise, the integration of the weak form needs to be done in the 
full multidimensional space and this requires an exponential number of operations. The separability of these operators is determined by the separability of the involved functions, namely: i) incident wave (5), ii) bathymetry coefficients (Remark 2), and iii) PML coefficients appearing in matrix $\mathbf{P}(8)$.

Separable versions of these functions might be obtained as a preprocess of the PGD algorithm via singular value decomposition or its higher-order extensions [36]. Here, an alternative procedure to find separable approximations of known functions based on the PGD method is proposed. It is called higher-order PGD-projection and formalized in Appendix A. In the case of using two separated functions, the PGD-projection provides an optimal decomposition coinciding with POD, and without the cost associated to an singular value decomposition (SVD) problem. This projection is particularly useful to reduce the rank of already separable functions as will be shown in the next Section. All the advantages of higher-order PGD-projection are discussed and demonstrated in Appendix A with several examples.

\subsection{Getting separable coefficients}

The separable version of the operator $A(\cdot, \cdot)$ is obtained by means of separable versions of the coefficients $b_{i}$ defined as follows,

$$
\begin{aligned}
a(u, \delta u ; \omega)=(\overbrace{k^{2} c c_{g}}^{b_{1}} s_{x} s_{y} u, \delta u)_{\Omega}-(\overbrace{c c_{g}}^{b_{2}} \mathbf{P} \nabla & \begin{aligned}
\nabla \delta u)_{\Omega} \\
+i \alpha\langle\underbrace{k c c_{g}}_{b_{3}} u, \delta u\rangle_{\Gamma_{\mathrm{R}}}
\end{aligned}+i\langle\underbrace{k c c_{g}}_{b_{3}} u, \delta u\rangle_{\Gamma_{\mathrm{pm} 1}} .
\end{aligned}
$$

The functions $1 / s_{x}$ and $1 / s_{y}$ that appear in $\mathrm{P} \mathbf{P}$, recall (8), need also a separable representation. Note that $s_{x}(x, \omega)=1+\sigma_{x}(x) / \omega$ and $s_{y}(y, \omega)=1+\sigma_{y}(y) / \omega$ are already separable.

By means of the PGD-projection proposed in Appendix A an optimal rank- $s_{i}$ separated representation of coefficient $b_{i}$ for $i=1,2,3$ is computed

$$
\pi^{\mathrm{pgd}}\left[b_{i}(h, \omega)\right]=\sum_{m=1}^{s_{i}} \beta_{i}^{m} B_{i, 1}^{m}(h) B_{i, 2}^{m}(\omega) .
$$

This representation uses normalized functions $B_{i, 1}^{m}$ and $B_{i, 2}^{m}$ and, consequently, $\beta_{i}^{m}$ provides information on the amplitude of term $m$. For instance, Figure 2 depicts for coefficient $b_{1}$ the normalized coefficients $\beta_{1}^{m} /\left\|\left[\beta_{1}^{1}, \ldots, \beta_{1}^{m}\right]\right\|_{2}$ for $m=1, \ldots, 30$. The range of bathymetry and frequency cover the range of realistic values on the Mediterranean coast. Only 20 terms are required to generate a very accurate separable structure. Similar results are observed for the other coefficients $b_{2}$ and $b_{3}$. 


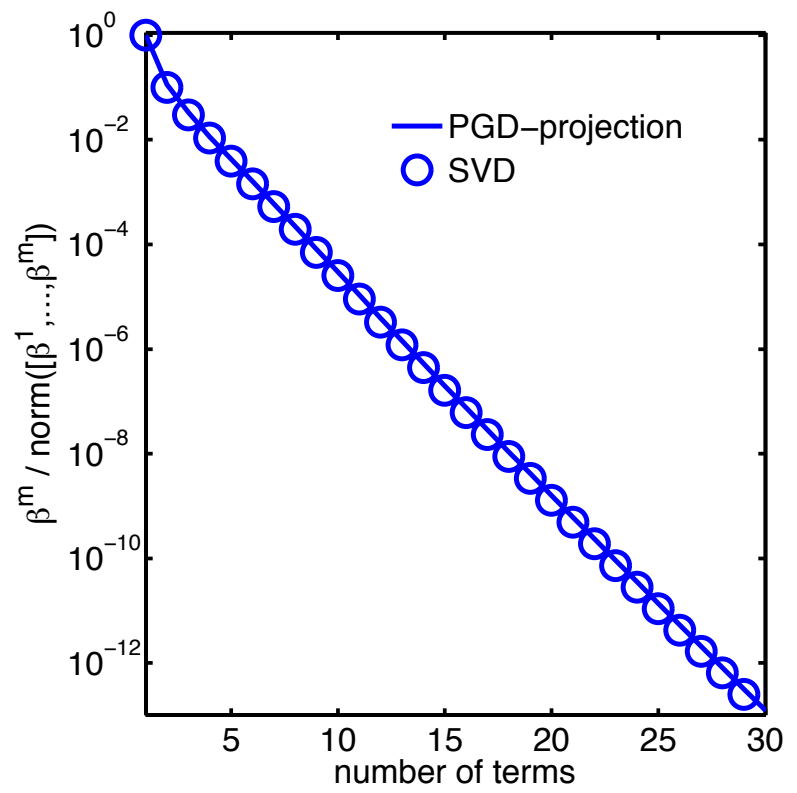

Figure 2: Normalized coefficients ( $\log _{10}$ scale) for the PGD-projection and SVD of $b_{1}(h, \omega)$. The range for the bathymetry and frequency are $h \in[1,65]$ and $\omega \in[0.4,2]$, both discretized with 1000 equidistant nodes.

The PGD-projection is also used to generate optimal separable forms for $1 / s_{x}(x, \omega)$ and $1 / s_{y}(y, \omega)$. This projection writes analogously to Eq. (19) using two separable functions with respect to $x$ (or $y$ ) in the first function and $\omega$ in the second one.

\subsection{Getting separable incident wave}

Once the separability of the bilinear form $A(\cdot, \cdot)$ is achieved, the linear functional $L(\cdot)$ is also separated provided a separable form of the incident wave, see Eq. (10b), is obtained.

In contrast to the expression of the MSE coefficients, the incident wave $u_{0}(x, y, \omega, \theta)$ depends on every parameter. Thus, an optimal decomposition is defined as

$$
\pi^{\mathrm{pgd}}\left[u_{0}(x, y, \omega, \theta)\right]=\sum_{m=1}^{q} \beta_{0}^{m} B_{0,1}^{m}(x, y) B_{0,2}^{m}(\omega, \theta),
$$

that separates $(x, y) \in \Omega_{\mathrm{pm} l}$ from the $2 \mathrm{D}$ parametric coordinate $(\omega, \theta) \in I_{\omega} \times I_{\theta}$. The cost of this projection is superior to the previous coefficients. Nevertheless, it is still affordable for small PML domains. Thanks to the spatially separated structure of $u_{0}$, recall Eq. (5), the expression (20) can be constructed as the product of: (i) the projection of $\exp \left(i k_{0} x \cos \theta\right)(1 \mathrm{D}$ in $x)$, and (ii) the projection of $\exp \left(i k_{0} y \sin \theta\right)(1 \mathrm{D}$ in $y)$. An example of these projections is shown in Appendix A.2. This procedure drastically reduces the cost for large PML domains. 
Remark 5 (practical computation of projections). Note that all the projections presented here, that separate both, coefficients and incident wave, can be computed in a proper reference domain and then mapped to the real coordinates. This reference domain must include all the possible combinations of the parameter values that are required in practice. Thus, there is no need to compute a new projection every time the bathymetry and the PML domain are modified.

\section{Application examples}

This section presents three problems: one academic example and two engineering applications related to harbor design. All problems are governed by the MSE (6) in an unbounded domain where the PML technique is applied in the outer boundary.

Before presenting the example in detail a few general remarks are pertinent. First, note that in order to ensure a correct absorption of the scattered waves, the width of the rectangular PML domain, namely $L_{\mathrm{pm} 1}$, is set as 1.5 times the maximum wave length induced by the lower frequency in each example [40]. Functions $s_{x}=1+\sigma_{x} / \omega$ and $s_{y}=1+\sigma_{y} / \omega$, which quantify the absorption of the scattered wave, are defined using a second order polynomial

$$
\sigma_{x}(x)=\sigma_{\max }\left(\left(x-x_{0}\right) / L_{\mathrm{pml}}\right)^{2} \quad\left(\text { analogously for } \sigma_{y}(y)\right),
$$

where $x_{0}$ (respectively $y_{0}$ ) stands for the coordinate value at which the interface boundary $\Omega_{\text {int }} \cap \Omega_{\mathrm{pml}}$ is placed. The maximum absorption $\sigma_{\max }$ is then set in order to maximize the damping of the scattered wave using the values proposed in [20].

Second, convergence criteria must be imposed. For the fixed point algorithms, see Eq. (15), convergence is assumed when

$$
\frac{\left\|\prod_{i=1}^{3} F_{i}^{(\nu)}-\prod_{i=1}^{3} F_{i}^{(\nu-1)}\right\|_{\mathcal{L}^{2}\left(\Omega \times I_{\omega} \times I_{\theta}\right)}^{2}}{\left\|\prod_{i=1}^{3} F_{i}^{(\nu)}\right\|_{\mathcal{L}^{2}\left(\Omega \times I_{\omega} \times I_{\theta}\right)}^{2}}<\varepsilon^{2},
$$

where $\nu$ stands for the nonlinear iteration counter (analogously for the auxiliary functions $\widetilde{F}_{i}$ of the PG PGD algorithm).

For the greedy procedure (the number of terms involved in the PGD solution) the stopping criteria is based on the contribution of the last term, namely

$$
\frac{\left\|F_{1} F_{2} F_{3}\right\|_{\mathcal{L}^{2}\left(\Omega \times I_{\omega} \times I_{\theta}\right)}^{2}}{\left\|u^{n-1}\right\|_{\mathcal{L}^{2}\left(\Omega \times I_{\omega} \times I_{\theta}\right)}^{2}}<\varepsilon^{2} .
$$

The condition (21) is straightforward to evaluate, it does not imply costly calculations and usually gives valid estimations for low tolerances (for instance $\varepsilon \leq 10^{-6}$ ). More accurate 
(and costly) criteria can be used to stop the enrichment procedure, for example using a goal-oriented strategy based on the solution of an adjoint problem, see [1].

A third point is how to evaluate the accuracy of the approximation provided by the PGD strategy. Here, two quantities of interest (QoI) related to harbor design are used. One is the wave height $H(x, y, \omega, \theta)=|u(x, y, \omega, \theta)|$, recall Remark 1. Other is the maximum wave height in an area of interest $\mathcal{A} \subset \Omega_{\text {int }}$ of the interior domain, that is

$$
H_{\max }(\omega, \theta)=\max _{(x, y) \in \mathcal{A}} H(x, y, \omega, \theta) .
$$

Based on these QoI, two error measures are defined: i) the normalized spatially-averaged error of the wave height at some design parameter values $\omega^{*}$ and $\theta^{*}$,

$$
\frac{\left\|H^{n}\left(x, y, \omega^{*}, \theta^{*}\right)-H\left(x, y, \omega^{*}, \theta^{*}\right)\right\|_{\mathcal{L}^{2}(\mathcal{A})}}{\left\|H\left(x, y, \omega^{*}, \theta^{*}\right)\right\|_{\mathcal{L}^{2}(\mathcal{A})}},
$$

and ii) the normalized parametrically-averaged error of the maximum wave height,

$$
\frac{\left\|H_{\max }^{n}(\omega, \theta)-H_{\max }(\omega, \theta)\right\|_{\mathcal{L}^{2}\left(I_{\omega} \times I_{\theta}\right)}}{\left\|H_{\max }(\omega, \theta)\right\|_{\mathcal{L}^{2}\left(I_{\omega} \times I_{\theta}\right)}} .
$$

The quantities $H^{n}$ and $H_{\max }^{n}$ are the measures based on the rank- $n$ PGD solution, while $H$ and $H_{\max }$ are based on a reference solution obtained numerically.

The fourth and last point concerns the numerical discretization. All the examples use standard continuous Galerkin finite elements. More precisely, the spatial meshes use fourth order simplices with a minimum wave resolution of 8 nodes per wavelength. Parametric meshes are all linear with a chebyshev distribution of the nodes.

\subsection{Scattering on a cylindrical obstacle}

The first example is a standard benchmark for scattering problems. It consists in a totally reflecting scatterer with radius $R=1$. The solution is parametrized in space and frequency, $u(x, y, \omega)$, leaving the incident angle fixed at value $\theta=3 \pi / 2$. The 3D PGD approximation is then given by

$$
u(x, y, \omega) \approx u^{n}(x, y, \omega)=\sum_{m=1}^{n} F_{1}^{m}(x, y) F_{2}^{m}(\omega) .
$$

Three different solutions are computed for three frequency regimes. Low frequencies: $I_{\omega}=$ [6.28, 10.47], medium: $I_{\omega}=[6.28,22.15]$ and high frequencies: $I_{\omega}=[6.28,31.45]$, with a minimum dimensionless wavenumber $k R=1$, and a maximum $k R=\{13,50,100\}$, respectively, on the cylindrical obstacle. In this first example a maximum number of 3 nonlinear iterations per term is used, recall Remark 4. The spatial domain, bathymetry and boundary conditions are shown in Figure 3 as well as the wave height field generated 

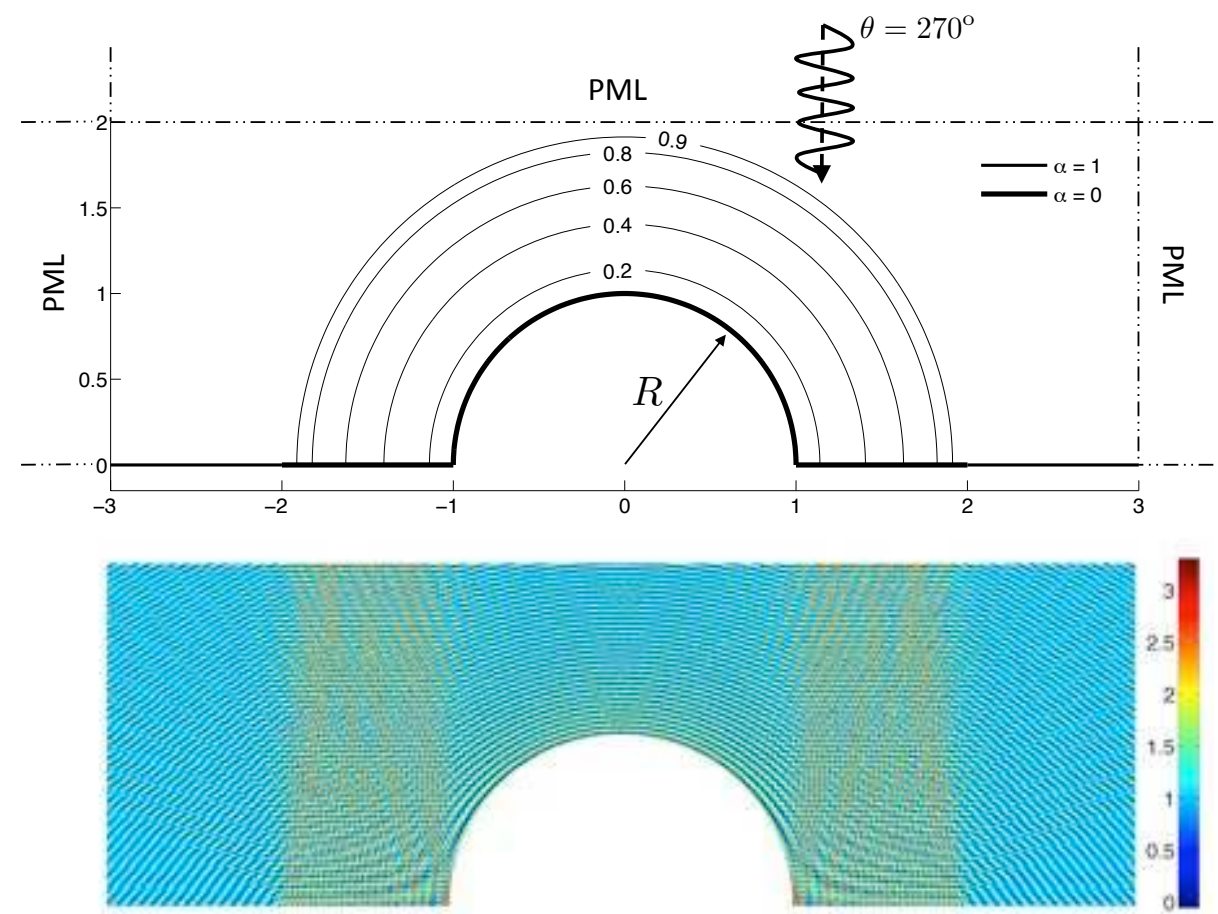

Figure 3: Cylindrical scattering: problem statement. Spatial domain with values of the absorbing coefficient $\alpha$ on the boundary and contour lines of the bathymetry (top). Waveheight for a fixed frequency $\omega^{*}=31.16 \mathrm{rad} / \mathrm{s}$ (bottom).
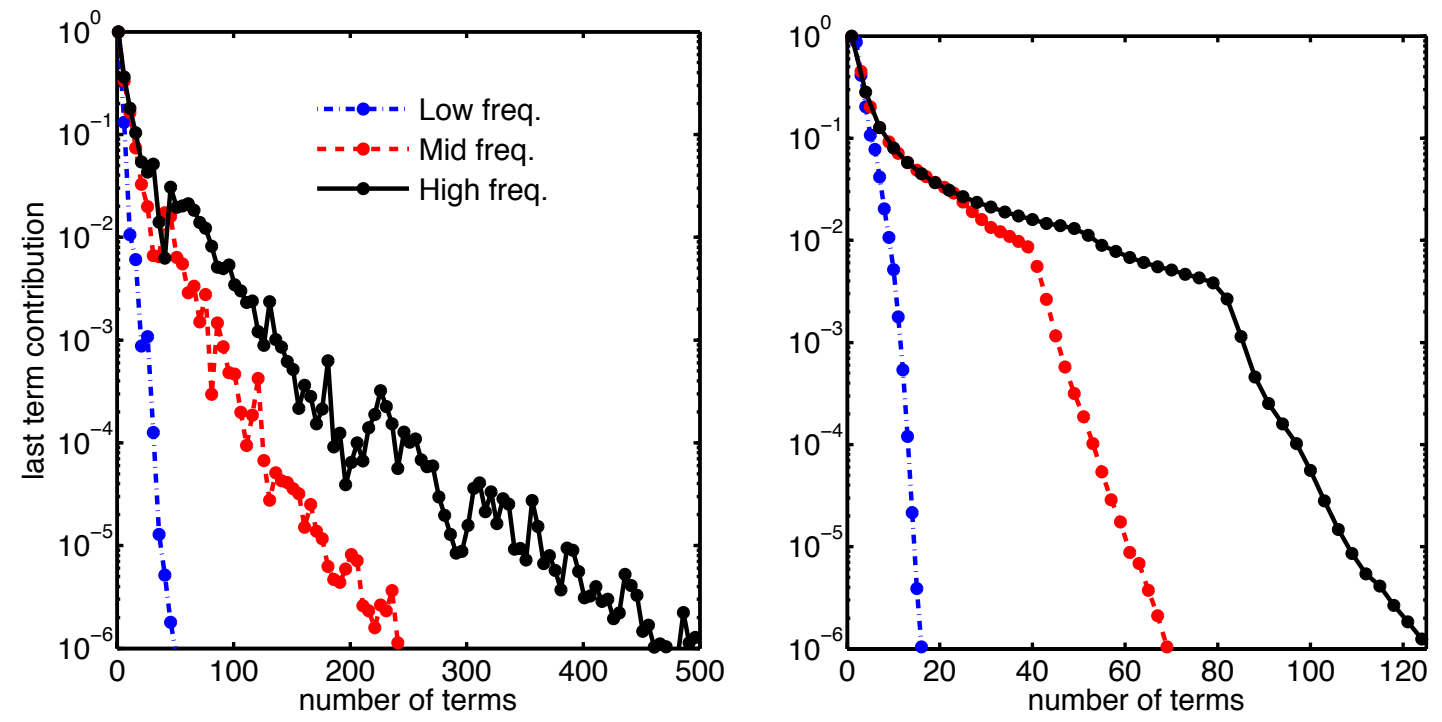

Figure 4: Cylindrical scattering: contribution of the last mode to the PG PGD (left) and its corresponding PGD-projection (right) for three different frequency ranges from low (maximum $k R=13$ ) to high (maximum $k R=100$ ). 
for the particular case $k R=99$. The fourth order spatial mesh has $\approx 4 \times 10^{5}$ nodes for the high frequency case, whereas the parametric domains, $I_{\omega}$, are discretized with an overkilled mesh of 1000 nodes.

Left panel of Figure 4 depicts the contribution of the last term, see Eq. (21), for the three solutions corresponding to the three frequency ranges. Only the PG PGD algorithm is depicted in this case. A strong influence on the convergence rate is observed when increasing the frequency range, being in agreement with previous applications of reduced modeling for scattering problems $[15,27]$. Moreover, note that convergence exhibits oscillations that tend to grow as the frequency range increases. This effect is largely amplified when using the standard PGD algorithm to approximate the solutions (not shown). Comparison of PGD algorithms is further presented for this example in terms of accuracy.

The right panel of Figure 4 shows the PGD-projected version of the three solutions. The PGD-projection procedure is equivalent to POD for two separated dimensions. The obtained solutions, therefore, are optimal in the number of terms (compare the $x$-axis of left and right panels of Figure 4). In addition the projected solutions present a monotone convergence. Note that, as the PGD-projection is applied to a separable function, its computational cost is very low compared with the PG PGD offline solution, see Appendix A. In addition, the large reduction in the number of terms contributes to reduce the time and memory required in the online phase.

The accuracy of (23) is studied, for a given frequency, using the first QoI-based error (22a). The area of interest in this case is the complete interior spatial domain. Three convergence curves are shown at each panel of Figure 5 corresponding to the online solutions given by the standard PGD algorithm, PG PGD and the PGD-projection. Recall that these errors are computed with real-time evaluations of the PGD. All depicted curves use fixed frequency values, namely $\omega^{*}$ in Figure 5, which do not coincide with the discretization of the parametric domain $I_{\omega}$. Note that results show a similar trend to that observed in the last term contribution of Figure 4: the larger the frequency ranges, the more terms are needed to obtain similar accuracy. Furthermore, small variations in frequency present in some cases very different convergence curves, as seen comparing the top and bottom rows of Figure 5. This variation in convergence curves is not present in the solutions provided by the PGD-projection.

Finally, it can be observed that, despite in this simple test the standard PGD algorithm converges, the PG PGD exhibits a better convergence.

\subsection{Mataró harbor}

The second example corresponds to a study of the water agitation in Mataró harbor, located North of Barcelona (Spain). In this case the realistic harbor geometry largely increases the number of reflected waves, increasing the difficulty of the computational problem. The computational domain, bathymetry and boundary conditions are shown in Figure 6 . The 

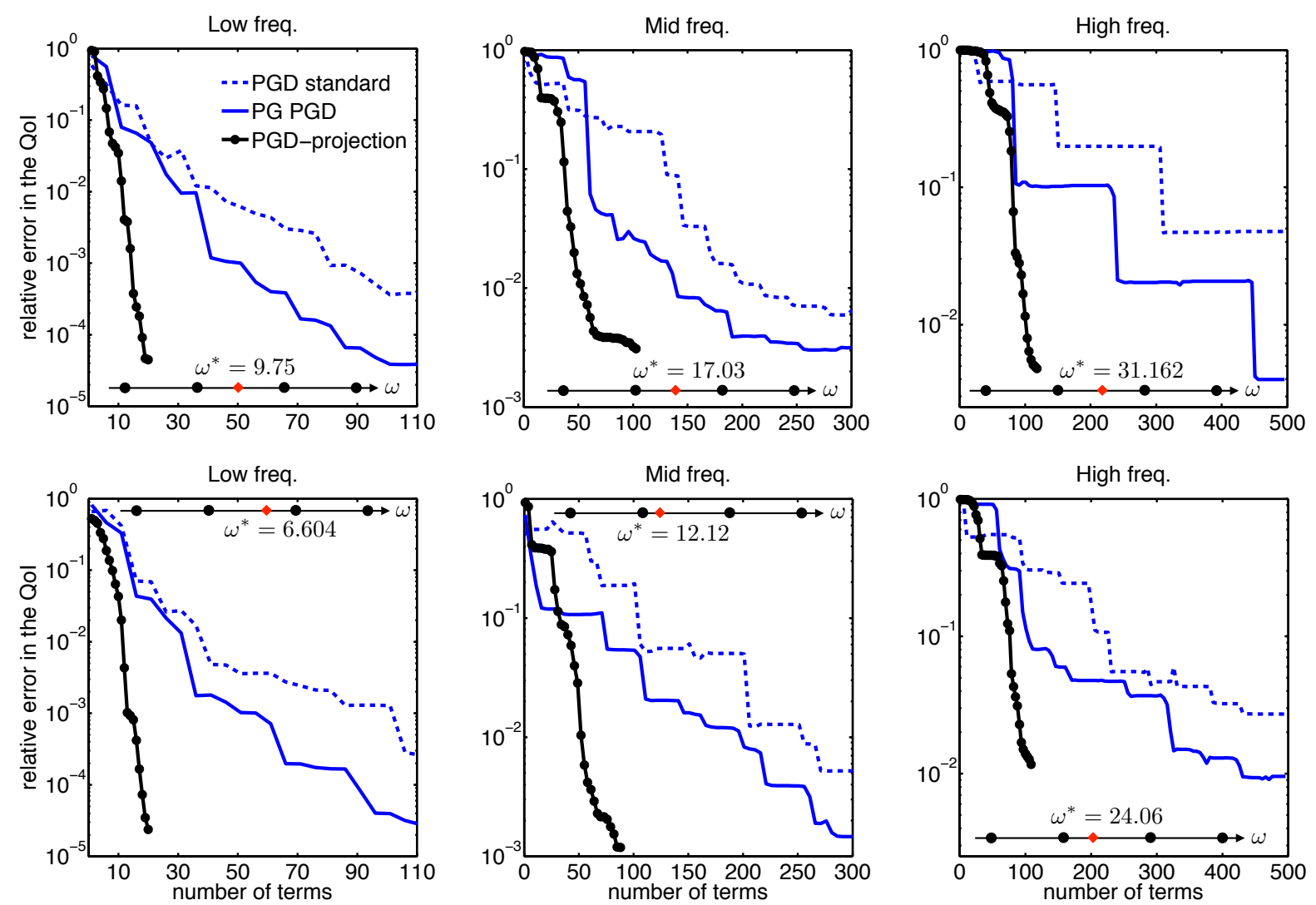

Figure 5: Cylindrical scattering: convergence of relative error of wave-height in the interior domain at a fixed frequency $\omega^{*}$, see Eq. (22a), versus number of modes. Plots depict three PGD strategies (PG PGD, standard and projection) for three frequencies ranges: low (left), medium (middle) and high (right). The relative position of value $\omega^{*}$ within the $1 \mathrm{D}$ range is highlighted by a red rhombus marker. 


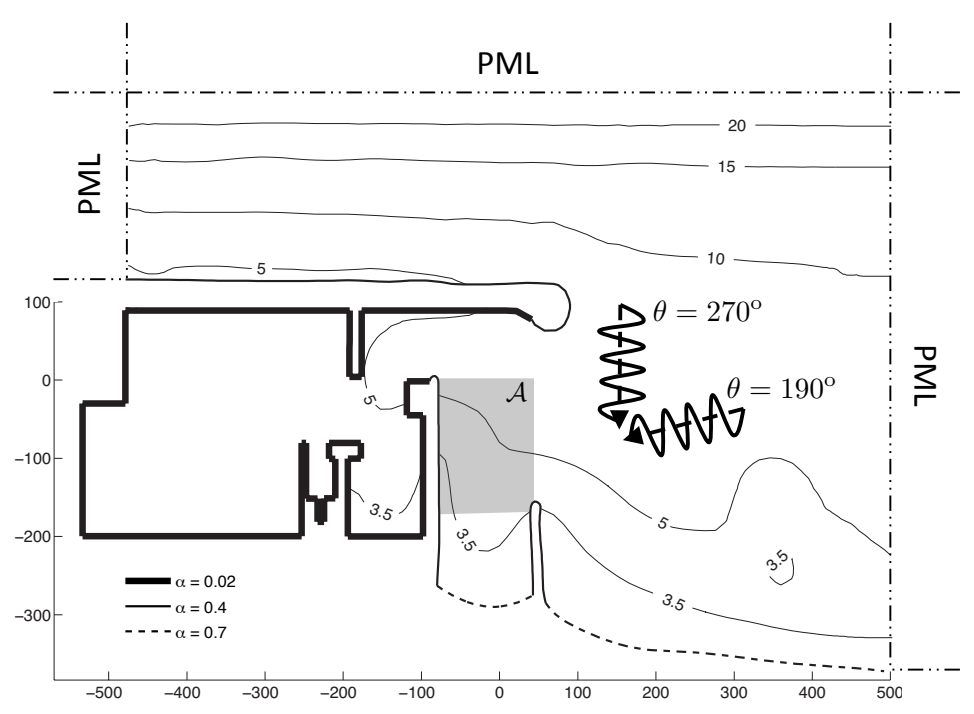

Figure 6: Mataró harbor: problem statement. Spatial domain with values of the absorbing coefficient $\alpha$ on the boundary and bathymetry. The area $\mathcal{A} \subset \Omega_{\text {int }}$ of interest is highlighted. The range of variability of the incoming wave direction is also illustrated.

area of interest $\mathcal{A} \subset \Omega_{\text {int }}$ defining the error measure (22) is also shown in the Figure. It corresponds to the wave impact region on the inlet channel of the harbor.

The solution in this case is fully parameterized with space, frequency and incoming direction, i.e. $u(x, y, \omega, \theta)$. Its PGD approximation, $u^{n}$, is separated in the form of Eq. (11). Incident waves are in accordance with those observed offshore in the region: $\omega \in$ $[0.39,0.63]$ (from $10 \mathrm{~s}$ to $16 \mathrm{~s}$ of wave period) and $\theta \in[1.05 \pi, 3 \pi / 2]$. The discretization used is $15757 \times 50 \times 50$ nodes for $(x, y), \omega$ and $\theta$ respectively. Using the notation introduced in Appendix A, the rank- $q$ projection of the PGD solution $u^{n}$ is computed here as

$$
u_{\pi}^{q}(x, y, \omega, \theta)=\pi^{\mathrm{pgd}}\left[u^{n}(x, y, \omega, \theta)\right]=\sum_{m=1}^{q} \beta^{m} F_{\pi, 1}^{m}(x, y) F_{\pi, 2}^{m}(\omega, \theta),
$$

and thus, optimal projections are obtained since $u_{\pi}^{q}$ is separated in two dimensions.

The contribution of the last term is compared in Figure 7 for the PG PGD, the standard PGD and the optimal PGD-projection. Despite this example remains in the low frequency regime, the standard PGD does not converge. In contrast, PG PGD is able to converge, but the number of terms required to reach a certain level of accuracy is far from optimal.

The computational cost of the offline phase is largely determined by the number of spatial problems to be solved (i.e. the number of iterations needed for convergence times the required terms). As commented earlier, the maximum number of nonlinear iterations per term, namely $\nu_{\max }$, has a direct impact on this cost. Its influence is explored here by solving the same problem for different values of $\nu_{\max }$ and measuring the error indicator $(22 \mathrm{~b})$ 


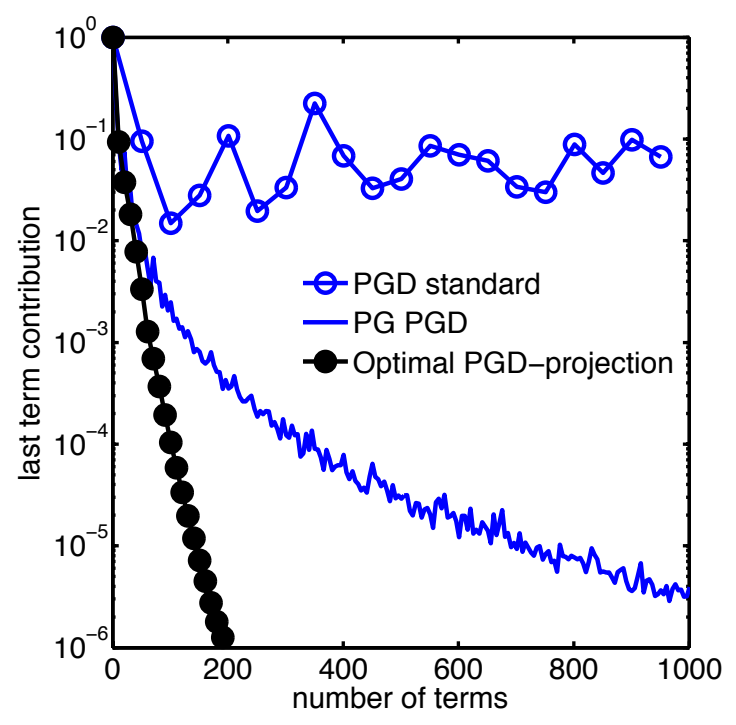

Figure 7: Mataró harbor: contribution of the last term to the standard PGD, PG PGD, and PGD-projected solution.
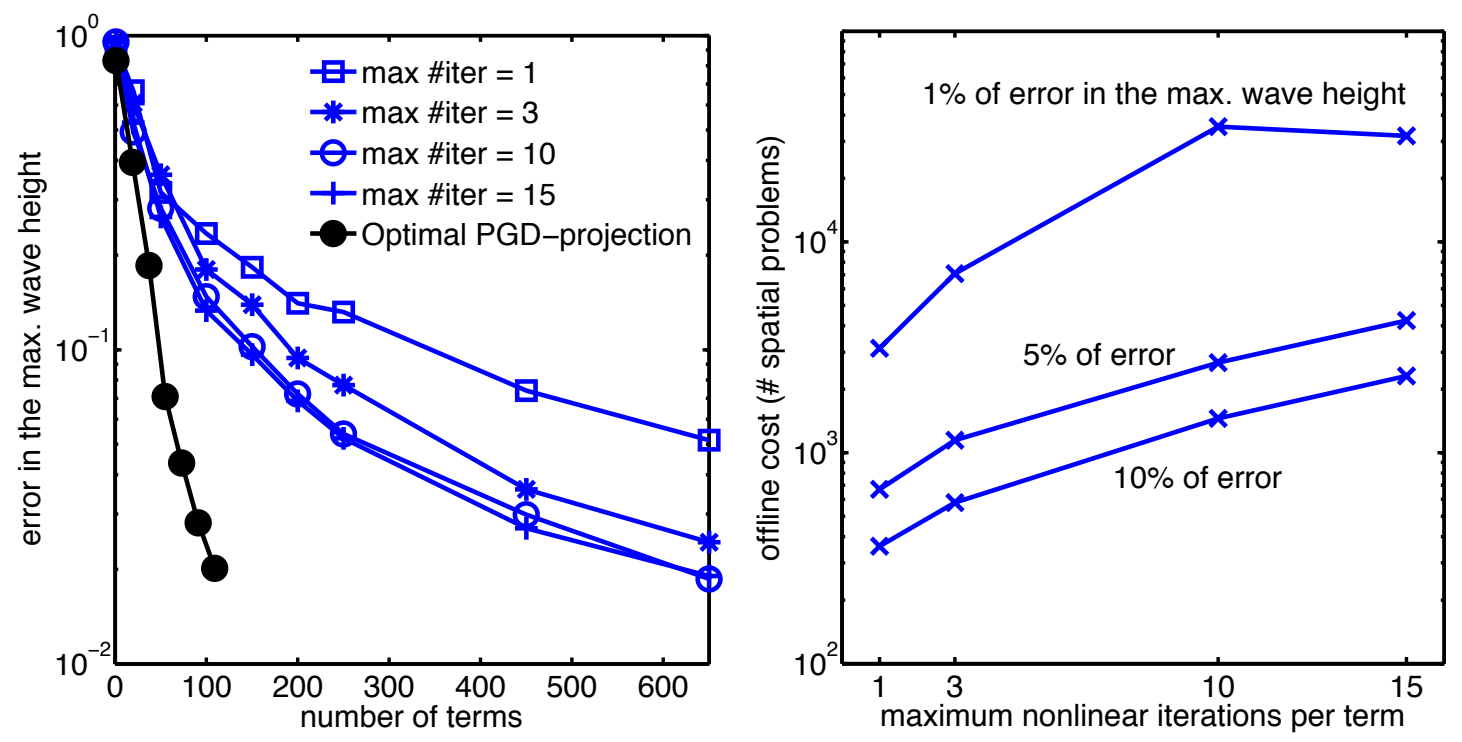

Figure 8: Mataró harbor: analysis of the maximum number of nonlinear PGD iterations $\nu_{\max }$. Error of the maximum wave height in the area of interest, see Eq. (22b), versus the number of terms of various PG PGD expansions (left). For fixed values of the error (22b), the required number of $2 \mathrm{D}$ problems solved in the PGD construction is shown versus the values of $\nu_{\max }$ (right). 

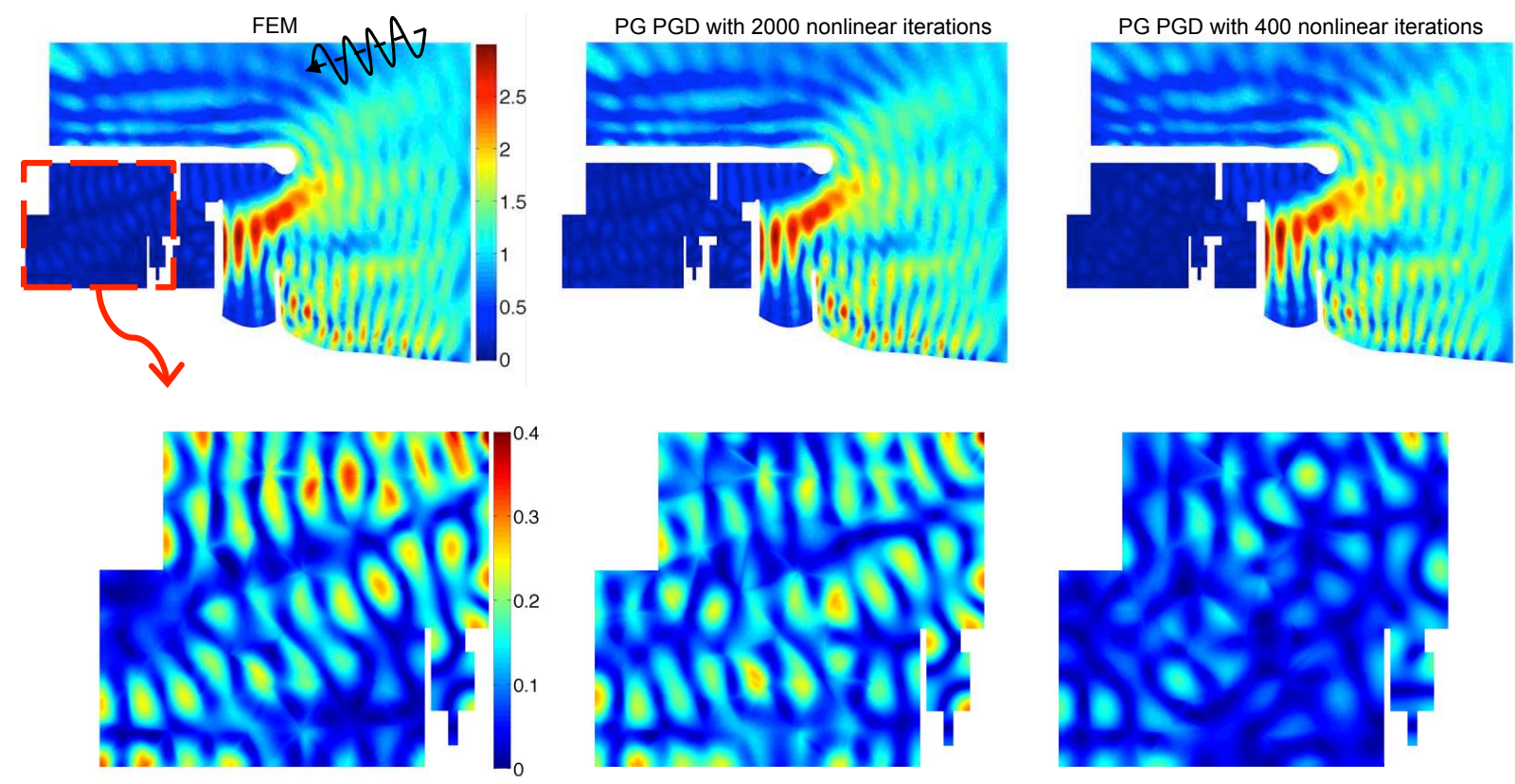

Figure 9: Mataró harbor: wave amplification for the particular case $\omega=0.61 \mathrm{rad} / \mathrm{s}$ and $\theta=194.5^{\circ}$. It is shown using spatial FEM (left column), PG PGD with 2000 nonlinear iterations (middle column) and PG PGD with 400 nonlinear iterations (right column). The interior area of the harbor is zoomed in the bottom row.

in the area $\mathcal{A}$. These tests (left panel of Figure 8) show that $\nu_{\max } Z 3$ does not improve the quality of the PGD solution significantly. Furthermore, the right panel of the same Figure depicts the offline cost (\# spatial problems) to reach a fixed level of error versus the value of $\nu_{\max }$. In this case, using the PGD-projected solution provided by the extreme case $\nu_{\max }=1$ gives the better performance in terms of accuracy and also in terms of computational cost.

Finally, a drastic increase on the computational cost is observed to reach an engineering accuracy in those areas where a lot of reflections are involved. This is shown in Figure 9 that depicts the wave amplification for an unfavorable propagation case. The spatial computation with FEM is used as a reference. Despite a good solution is predicted in the exterior harbor region with 400 total nonlinear iterations, at least 5 times more terms are required to capture the wave physics in the interior (much more reflective area).

\subsection{Barcelona harbor}

A problem similar to the previous example is solved now for the Barcelona harbor. In this case the geometry is more complex and the size of the harbor is larger, further increasing the number of reflections and therefore making the problem more challenging. The spatial domain, bathymetry and boundary conditions are depicted in Figure 10. The incoming direction $\theta \in[\pi, 3 \pi / 2]$ is considered, and the frequency range is $\omega \in[0.39,0.63]$ (same 


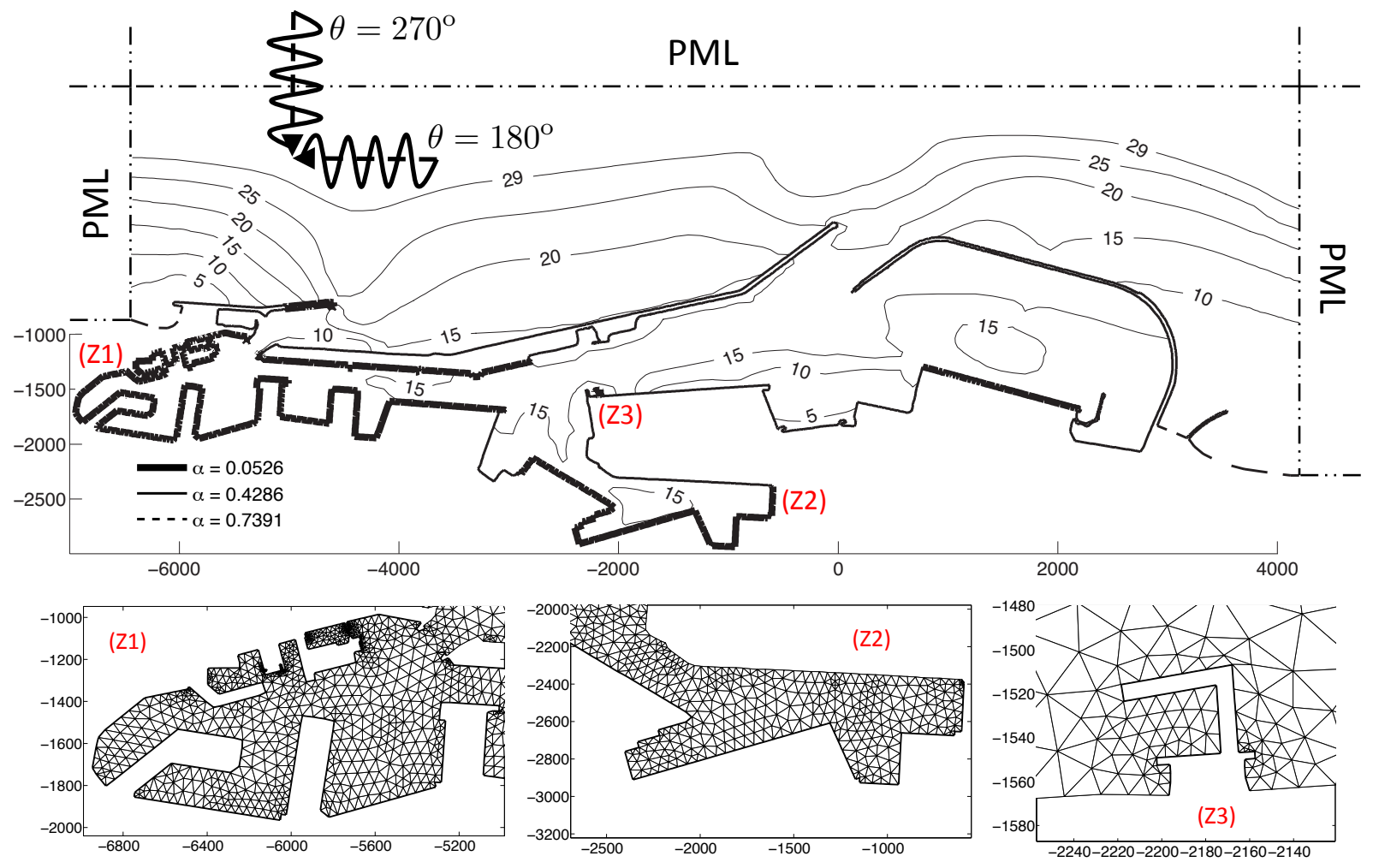

Figure 10: Barcelona harbor: problem statement. Spatial domain with values of the absorbing coefficient $\alpha$ on the boundary, see Eq. (3), and contour lines of the bathymetry. The range of variability of the incoming wave direction is also illustrated. The spatial mesh is shown for three different zooms of the interior domain.

as in the previous example as both locations are close by). Nevertheless, the maximum frequency induces here approximately 90 waves within the domain, moving the study from low to medium-high frequency range. The discretization has $210^{5} \times 100 \times 50$ number of nodes for $(x, y), \omega$ and $\theta$ respectively. Optimal PGD-projected solutions are also computed analogously to Eq. (24).

The Figure 11 depicts the contribution of the last term to the PG PGD and its optimal PGD-projection. Standard PGD is in this case discarded. The convergence of PG PGD is slow, nevertheless, in the first 500 terms it concedes with the optimal curve, showing the proper behavior of the algorithm (these terms provide relevant information) and the inherent complexity of the problem.

The accuracy of the PG PGD is studied in three different areas of interest, see Figure 12. In each area the PGD solution is compared with a reference one (computed using standard FEM) for a given value of the parameters. Good results, always under engineering accuracy, are observed in all cases and specially for the wave phase.

The PG PGD provided 4015 terms that were later PGD-projected to the 1500 optimal 


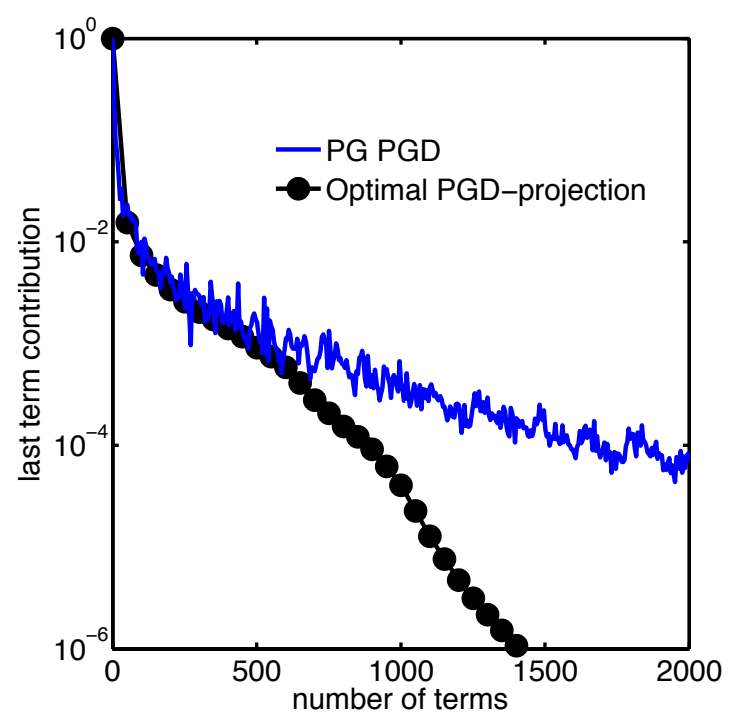

Figure 11: Barcelona harbor: Contribution of the last term $\left(\log _{10}\right.$ scale) to the PG PGD and to its PGD-projected solution.
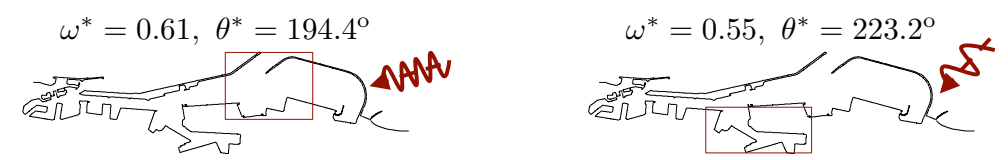

$$
\omega^{*}=0.42, \theta^{*}=194.4^{\circ}
$$
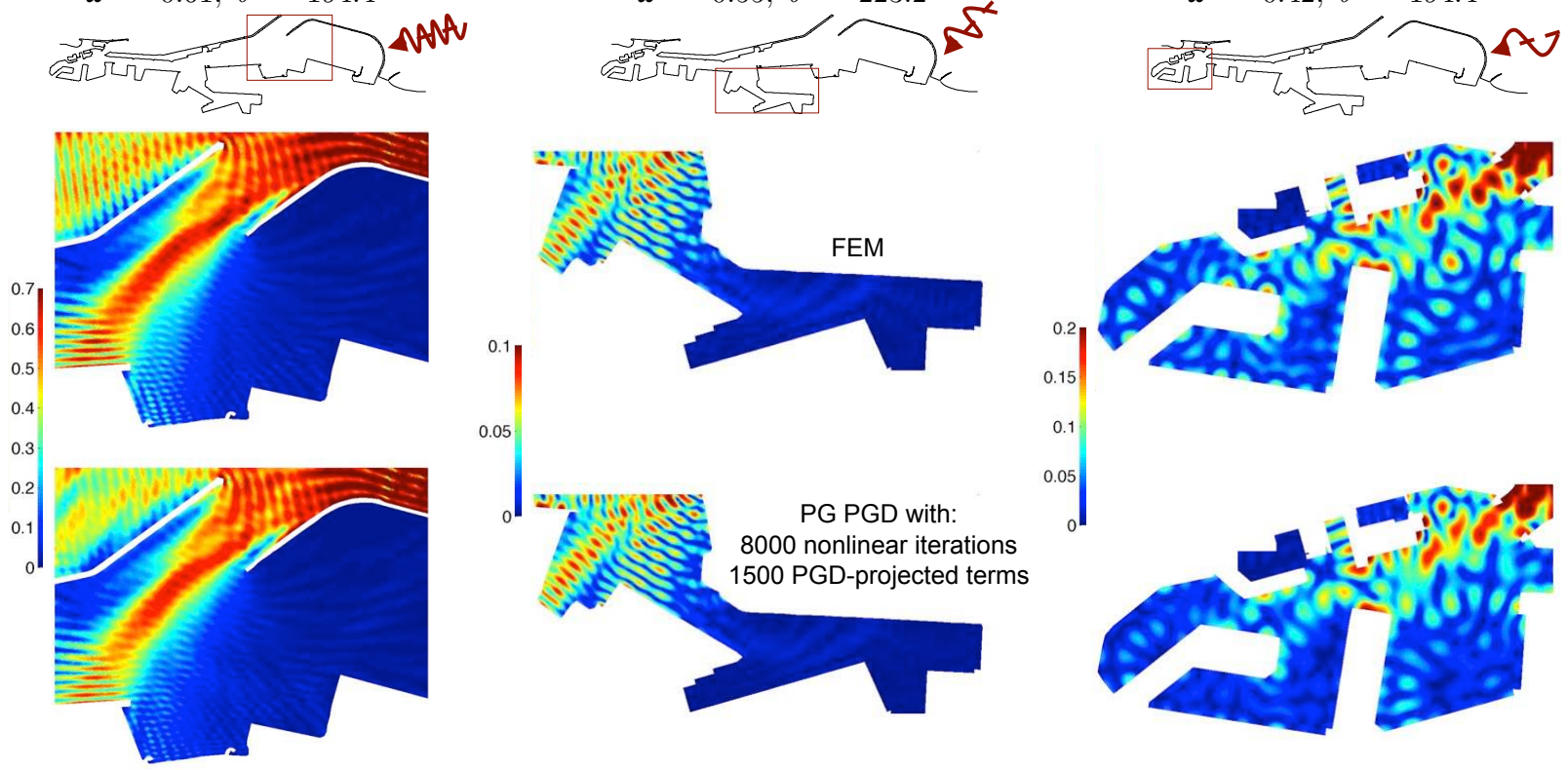

Figure 12: Barcelona harbor: wave-height in different harbor areas (highlighted on top) and values of $\left(\omega^{*}, \theta^{*}\right)$ : short waves with $(0.61,1.08 \pi)$ (left), mid waves with $(0.55,1.24 \pi)$ (middle) and long waves with $(0.42,1.08 \pi)$ (right). The spatial FEM solution (top) and the PG PGD interpolated solution (bottom) with 1500 PGD-projected terms (8000 solves) are shown. 
terms shown in the Figure 12. The maximum wave height along the inlet channel of the harbor (left column of the same Figure) is well-captured with less than $10 \%$ of error. Same behavior is observed for the inner harbor region (in the middle column). For the more reflective area (right column) the error increases up to the $15 \%$. This corroborates the results of the previous example, where the required terms drastically increase in presence of higher reflections.

\section{Concluding remarks}

This paper proposes the application of PGD to approximate the 4D generalized solution of the Helmholtz equation in heterogeneous and unbounded domains. The generalization includes variability of some design parameters: frequency and incoming wave direction. Particularly, the propagation of sea waves is considered and the harbor agitation study is used as an application example. Each direct computation of the problem involves spatial and parameter dependent coefficients, unbounded physical domains and large reflections induced by the complex geometry. Moreover, the practical applications usually impose numerous direct solutions of this problem for different values of the design parameters.

The non-separability issue of the presented problem is solved using a higher-order PGDprojection: an a posteriori use of the PGD method that separates known multidimensional functions. Formalization of the problem and comparison examples are provided. Results show that optimal expansions (equivalent to those from POD) are obtained when two separated dimensions are used. When the separation is done in more that two dimensions, the PGD-projection can outperform decompositions given by the standard higher-order singular value decomposition (HOSVD). Moreover, the projection of previously computed PGD solutions provides a drastic reduction in the number of terms of the expansion with marginal extra cost. Thus, improvements in memory requirements and online runtime are obtained.

Two different PGD approaches, standard and Petrov-Galerkin ones, are compared. The PG PGD clearly outperforms the standard one, providing faster convergences, and converging where the standard PGD fails. Furthermore, the approach requires only a few nonlinear iterations per term $(\leq 3)$ in the offline PGD constructor.

The engineering study of water agitation in harbors for multiple and fast queries can be efficiently performed via the numerical techniques presented in this work. However, a high frequency range and a large number of reflections degrade the convergence of algorithms. For more general Helmholtz problems, improvements on the PGD are still needed in such situations if higher accuracy is of concern. 


\section{Acknowledgements}

This work has been partially supported by the Spanish Ministry of Science and Competitiveness, through grant number CICYT-DPI2011-27778-C02-01/02 and by the Generalitat de Catalunya, grant number 2014-SGR-1471.

\section{References}

[1] A. Ammar, F. Chinesta, P. Díez, and A. Huerta. An error estimator for separated representations of highly multidimensional models. Comput. Methods Appl. Mech. Eng., 199:1872-1880, 2010.

[2] A. Ammar, B. Mokdad, F. Chinesta, and R. Keunings. A new family of solvers for some classes of multidimensional partial differential equations encountered in kinetic theory modelling of complex fluids. J. Non-Newtonian Fluid Mech., 139:153-176, 2006.

[3] A. Ammar, B. Mokdad, F. Chinesta, and R. Keunings. A new family of solvers for some classes of multidimensional partial differential equations encountered in kinetic theory modeling of complex fluids. Part II: transient simulation using space-time separated representations. J. Non-Newtonian Fluid Mech., 144:98-121, 2007.

[4] A. Ammar, D. Ryckelynck, F. Chinesta, and R. Keunings. On the reduction of kinetic theory models related to finitely extensible dumbbells. J. Non-Newtonian Fluid Mech., 134(1-3 SPEC. ISS.):136-147, 2006.

[5] D. Amsallem and C. Farhat. Interpolation method for adapting reduced-order models and application to aeroelasticity. AIAA J., 46(7):1803-1813, 2008.

[6] C. A. Andersson and R. Bro. The N-way Toolbox for MATLAB. Chemom. Intell. Lab. Syst., 52(1):1-4, 2000.

[7] I. Babuška, F. Ihlenburg, E. Paik, and S. Sauter. A Generalized Finite Element Method for solving the Helmholtz equation in two dimensions with minimal pollution. Comput. Methods Appl. Mech. Eng., 128(3-4):325-359, 1995.

[8] U. Basu and A. K. Chopra. Perfectly matched layers for time-harmonic elastodynamics of unbounded domains: Theory and finite-element implementation. Comput. Methods Appl. Mech. Eng., 192(11-12):1337-1375, 2003.

[9] J.-P. Berenger. A perfectly matched layer for the absorption of electromagnetic waves. J. Comput. Phys., 114(2):185-200, 1994.

[10] H. Bériot, G. Gabard, and E. Perrey-Debain. Analysis of high-order finite elements for convected wave propagation. Int. J. Numer. Methods Eng., 96(11):665-688, 2013. 
[11] J. C. W. Berkhoff. Computation of combined refraction-diffraction. In Proc. 13th Coastal Engineering Conference, volume 1, pages 471-490, Vancouver, Canada, 1972.

[12] G. Berkooz, P. Holmes, and J. L. Lumley. The proper orthogonal decomposition in the analysis of turbulent flows. Annu. Rev. Fluid Mech., 25(1):539-575, 1993.

[13] A. Bermúdez, L. Hervella-Nieto, A. Prieto, and R. Rodríguez. An exact bounded perfectly matched layer for time-harmonic scattering problems. SIAM J. Sci. Comput., 30(1):312-338, 2007.

[14] J. D. Carroll and J.-J. Chang. Analysis of individual differences in multidimensional scaling via an n-way generalization of "Eckart-Young" decomposition. Psychometrika, 35(3):283-319, 1970.

[15] Y. Chen, J. S. Hesthaven, Y. Maday, J. Rodrguez, and X. Zhu. Certified reduced basis method for electromagnetic scattering and radar cross section estimation. Comput. Methods Appl. Mech. Eng., 233-236:92-108, 2012.

[16] F. Chinesta, A. Ammar, and E. Cueto. Proper generalized decomposition of multiscale models. Int. J. Numer. Methods Eng., 83(8-9):1114-1132, 2010.

[17] F. Chinesta, A. Ammar, F. Lemarchand, P. Beauchene, and F. Boust. Alleviating mesh constraints: Model reduction, parallel time integration and high resolution homogenization. Comput. Methods Appl. Mech. Eng., 197(5):400-413, 2008.

[18] F. Chinesta, P. Ladeveze, and E. Cueto. A short review on model order reduction based on proper generalized decomposition. Arch. Comput. Methods Eng., 18(4):395404, 2011.

[19] F. Chinesta, A. Leygue, F. Bordeu, J. Aguado, E. Cueto, D. Gonzalez, I. Alfaro, A. Ammar, and A. Huerta. PGD-Based Computational Vademecum for Efficient Design, Optimization and Control. Arch. Comput. Methods Eng., 20(1):31-59, 2013.

[20] F. Collino and P. Monk. Optimizing the perfectly matched layer. Comput. Methods Appl. Mech. Eng., 164(1-2):157-171, 1998.

[21] L. De Lathauwer, B. De Moor, and J. Vandewalle. A multilinear singular value decomposition. SIAM J. Matrix Anal. Appl., 21(4):1253-1278, 2000.

[22] E. Demaldent and S. Imperiale. Perfectly matched transmission problem with absorbing layers: Application to anisotropic acoustics in convex polygonal domains. Int. J. Numer. Methods Eng., 96(11):689-711, 2013.

[23] L. Demkowicz and J. Shen. A few new (?) facts about infinite elements. Comput. Methods Appl. Mech. Eng., 195(29-32):3572-3590, 2006. 
[24] A. Deraemaeker, I. Babuška, and P. Bouillard. Dispersion and pollution of the FEM solution for the Helmholtz equation in one, two and three dimensions. Int. J. Numer. Methods Eng., 46(4):471-499, 1999.

[25] T. H. Fay. The butterfly curve. Am. Math. Mon., 96(5):pp. 442-443, 1989.

[26] G. J. Fix and S. P. Marin. Variational methods for underwater acoustic problems. J. Comput. Phys., 28(2):253-270, 1978.

[27] M. Ganesh, J. S. Hesthaven, and B. Stamm. A reduced basis method for electromagnetic scattering by multiple particles in three dimensions. J. Comput. Phys., 231(23):7756-7779, 2012.

[28] G. Giorgiani, D. Modesto, S. Fernández-Méndez, and A. Huerta. High-order continuous and discontinuous Galerkin methods for wave problems. Int. J. Numer. Methods Fluids, 73(10):883-903, 2013.

[29] D. Givoli. Recent advances in the DtN FE Method. Arch. Comput. Methods Eng., 6(2):71-116, 1999.

[30] D. Givoli. High-order local non-reflecting boundary conditions: A review. Wave Motion, 39(4):319-326, 2004.

[31] D. González, A. Ammar, F. Chinesta, and E. Cueto. Recent advances on the use of separated representations. Int. J. Numer. Methods Eng., 81(5):637-659, 2010.

[32] R. A. Harshman. Foundations of the PARAFAC procedure: Models and conditions for an "explanatory" multimodal factor analysis. UCLA working papers in phonetics, 16:1-84, 1970.

[33] F. Ihlenburg and I. Babuška. Finite element solution of the Helmholtz equation with high wave number Part I: The h-version of the FEM. Comput. Math. Appl., 30(9):9-37, 1995.

[34] F. Ihlenburg and I. Babuška. Finite element solution of the Helmholtz equation with high wave number Part II: the h-p version of the FEM. SIAM J. Numer. Anal., 34(1):315-358, 1997.

[35] K. Karhunen. Uber lineare methoden in der wahrscheinlichkeitsrechnung. Ann. Acad. Sci. Fenn., 37:1-79, 1946.

[36] T. G. Kolda and B. W. Bader. Tensor decompositions and applications. SIAM Rev., 51(3):455-500, 2009.

[37] P. Ladevèze, J.-C. Passieux, and D. Néron. The latin multiscale computational method and the proper generalized decomposition. Comput. Methods Appl. Mech. Eng., 199(21-22):1287-1296, 2010. 
[38] M. Loève. Probability Theory. Van Nostrand Princeton, third edition, 1963.

[39] A. Loula and D. Fernandes. A quasi optimal Petrov-Galerkin method for Helmholtz problem. Int. J. Numer. Methods Eng., 80(12):1595-1622, 2009.

[40] C. Michler, L. Demkowlcz, J. Kurtz, and D. Pardo. Improving the performance of perfectly matched layers by means of hp-adaptivity. Numer. Meth. Part. Differ. Equ., 23(4):832-858, 2007.

[41] A. K. Noor and J. M. Peters. Reduced basis technique for nonlinear analysis of structures. AIAA J., 18(4):455-462, 1980.

[42] A. Nouy. A generalized spectral decomposition technique to solve a class of linear stochastic partial differential equations. Comput. Methods Appl. Mech. Eng., 196:4521-4537, 2007.

[43] A. Nouy. A priori model reduction through proper generalized decomposition for solving time-dependent partial differential equations. Comput. Methods Appl. Mech. Eng., 199:1603-1626, 2010.

[44] P. Rouch and P. Ladevèze. The variational theory of complex rays: A predictive tool for medium-frequency vibrations. Comput. Methods Appl. Mech. Eng., 192(2830):3301-3315, 2003.

[45] R. Sevilla, S. Fernández-Méndez, and A. Huerta. NURBS-Enhanced Finite Element Method (NEFEM). Int. J. Numer. Methods Eng., 76(1):56-83, 2008.

[46] R. Sevilla, S. Fernandez-Mendez, and A. Huerta. NURBS-Enhanced Finite Element Method (NEFEM) a seamless bridge between CAD and FEM. Arch. Comput. Methods Eng., 18(4):441-484, 2011.

[47] I. Singer and E. Turkel. A perfectly matched layer for the Helmholtz equation in a semi-infinite strip. J. Comput. Phys., 201(2):439-465, 2004.

[48] L. L. Thompson and P. M. Pinsky. Galerkin least-squares finite element method for the two-dimensional Helmholtz equation. Int. J. Numer. Methods Eng., 38(3):371-397, 1995.

[49] S. Tsynkov. Numerical solution of problems on unbounded domains. A review. Appl. Numer. Math., 27(4):465-532, 1998.

[50] A. Vion, R. Sabariego, and C. Geuzaine. A model reduction algorithm for solving multiple scattering problems using iterative methods. IEEE Trans. Magn., 47(5):14701473, 2011. 


\section{A The higher-order PGD-projection}

This Appendix introduces a method that uses the PGD rationale to obtain separable approximations of known functions. Similarly to higher-order SVD [21] or the so-called CANDECOMP/PARAFAC (CP) methods [14, 32], this approach is designed to compute separable multidimensional functions. For a comprehensive review in HOSVD and CP methods see [36]. The PGD-projection generally produces separable representations with less terms compared with HOSVD. Moreover, the projection does not require the a priori selection of the number of terms for the separated solution (as CP does).

Consider a known $d$-dimensional function $f\left(z_{1}, \ldots, z_{d}\right)$ with coordinates $z_{i} \in \Omega_{i}$ for $i=1, \ldots, d$, which can be evaluated at any point of the high-dimensional domain $\Omega=$ $\Omega_{1} \times \cdots \times \Omega_{d}$. A rank-n PGD approximation of $f$ is defined as

$$
\begin{aligned}
f_{\pi}^{n}\left(z_{1}, \ldots, z_{d}\right)=\pi^{\mathrm{pgd}}\left[f\left(z_{1}, \ldots, z_{d}\right)\right] & =\sum_{m=1}^{n} \beta^{m} \prod_{i=1}^{d} F_{\pi, i}^{m}\left(z_{i}\right) . \\
& =f_{\pi}^{n-1}\left(z_{1}, \ldots, z_{d}\right)+\beta^{n} \prod_{i=1}^{d} F_{\pi, i}\left(z_{i}\right) .
\end{aligned}
$$

The coefficients $\beta^{m}$ are determined by a $\mathcal{L}^{2}$ projection once all $F_{\pi, i}^{m}$ are known (note that they are normalized, i.e. $\left\|F_{\pi, i}^{m}\right\|_{\Omega_{i}}=1$ for $m=1, \ldots, n$ and $i=1, \ldots, d$ ). This implies solving the typical symmetric and dense system of normal equations

$$
\sum_{m=1}^{n}\left(\psi_{s}, \psi_{m}\right)_{\Omega} \beta^{m}=\left(\psi_{s}, f\right)_{\Omega} \text { for all } s=1, \ldots, n
$$

with $\psi_{s}=\prod_{i=1}^{d} F_{\pi, i}^{s}$. While the rhs requires to integrate over the $d$-dimensional domain, the coefficients of the lhs matrix are simply products of 1D integrals, that is

$$
\left(\psi_{s}, \psi_{m}\right)_{\Omega}=\prod_{i=1}^{d}\left(F_{\pi, i}^{s}, F_{\pi, i}^{m}\right)_{\Omega_{i}}
$$

The greedy algorithm described in Section 4.1 with an alternating direction approach is used to compute functions $F_{\pi, i}^{m}$ for $m=1, \ldots, n$ and $i=1, \ldots, d$. This strategy pursues finding the separable approximation defined by (25) that minimizes the $\mathcal{L}^{2}$ distance between $f_{\pi}^{n}$ and $f$. However, as it will be explained later, it only guarantees to find the optimum when separating two dimensions. Each term ("mode") is obtained with the $\mathcal{L}^{2}$ projection on the tangent space, namely

$$
\left(\delta f, \prod_{i=1}^{d} F_{\pi, i}\right)_{\Omega}=\left(\delta f, f-f_{\pi}^{n-1}\right)_{\Omega}
$$


with test functions in the tangent space

$$
\delta f=\delta F_{\pi, 1} F_{\pi, 2} \cdots F_{\pi, d}+F_{\pi, 1} \delta F_{\pi, 2} \cdots F_{\pi, d}+\cdots+F_{\pi, 1} \cdots F_{\pi, d-1} \delta F_{\pi, d},
$$

and then normalized.

The following examples show the behavior and properties of the PGD-projection. When the separation involves two dimensions only, the PGD-projection minimizes the L2 distance in the same way POD does. The two separation procedures are therefore identical in that case and the separated solution obtained by PGD is optimal. This result coincides with [43]. Note however, that, in contrast with POD, the PGD-projection does not requires the solution of a SVD problem. Moreover, with PGD the approximation space for the separated representation is taken into account during the minimization process. Consequently, there is no need for extra interpolation techniques at those values outside the snapshots space, see for instance [5]. Furthermore, PGD has the advantage of a straightforward generalization to higher dimensions. There is no need for special implementations such as in HOSVD, and in the tested examples, PGD produces lower rank solution compared to HOSVD to obtain a given accuracy.

Finally, a practical use of the PGD-projection concerns the compression (reduction in the number of terms) of an already separated function $f$. This process is extremely fast when implementing the PGD-projection. The rhs of (26), when $\mathrm{f}$ is separable, is computed as products of 1D integrals as discussed in Section 5, therefore this compression is fast to perform. Furthermore, these integrals are all $\mathcal{L}^{2}$-projections on both sides of Eq. (26), implying that the computation of $F_{\pi, i}$ for $i=1, \ldots, d$ is performed by solving a diagonal linear system. The efficiency of the algorithm is therefore drastically improved.

These properties are shown next using three different examples. All the PGD-projections are computed with a maximum number of 5 iterations per term. For comparison purposes, the HOSVD is also computed in the last example using the extended N-way package for tensor decomposition in MATLAB ${ }^{\circledR}[6]$.

\section{A.1 Reproducing a separable function}

A separable function $f(x, y)$ with $(x, y) \in[0,1]^{2}$ is considered first. It consists in the product of two 1D polynomials, namely $P_{q}(x)$ and $P_{s}(y)$, defined by

$$
f(x, y)=P_{q}(x) P_{s}(y)=\sum_{i=0}^{q} x^{i} \sum_{j=0}^{s} y^{j}=1+x+y+x y+\cdots+x^{q} y^{s} .
$$

Note that the actual rank of this function is one. Therefore the PGD-projection, namely

$$
f_{\pi}^{n}(x, y)=\pi^{\mathrm{pgd}}[f(x, y)]=\sum_{m=1}^{n} \beta^{m} F_{\pi, 1}^{m}(x) F_{\pi, 2}^{m}(y)
$$




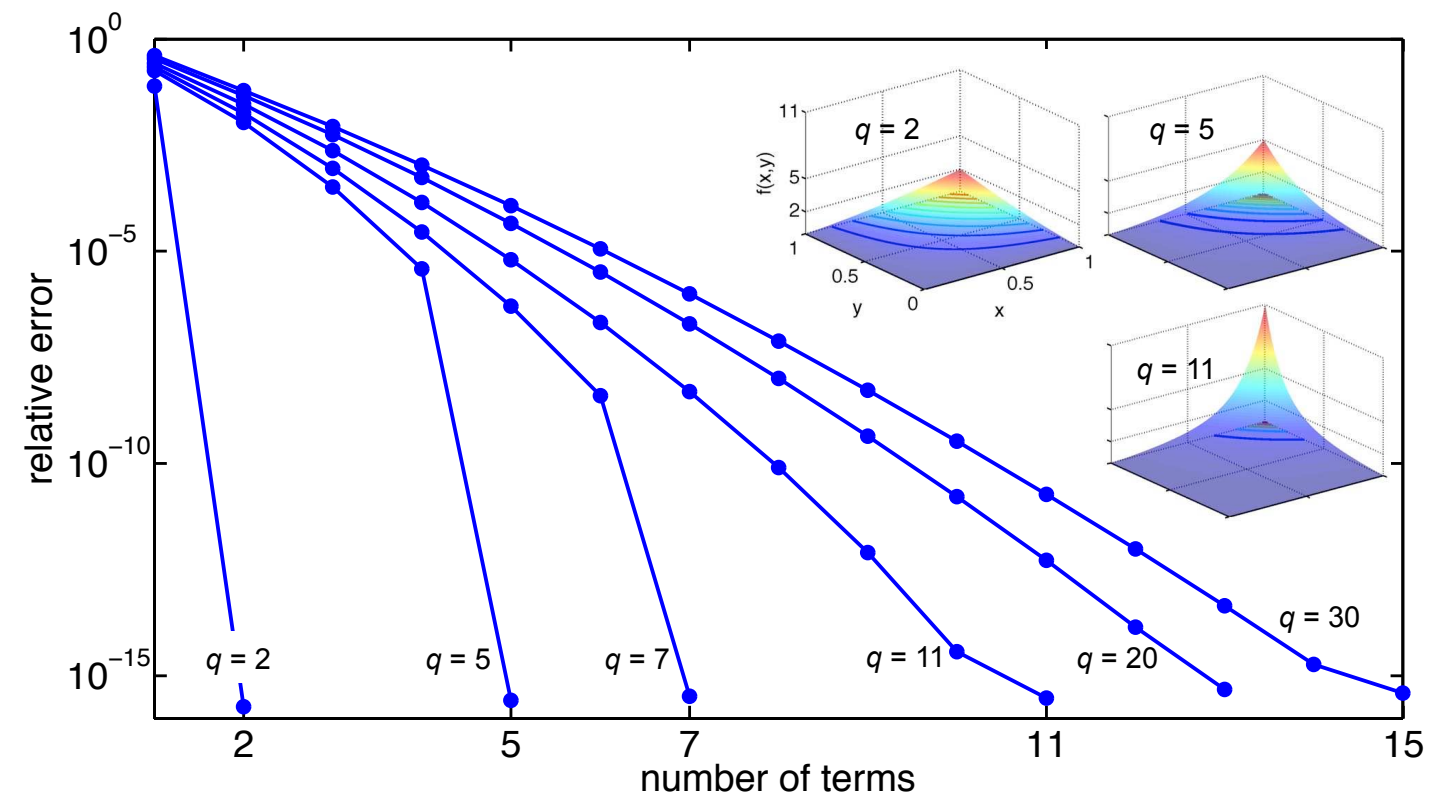

Figure 13: Separable function: convergence of the $\mathcal{L}^{2}$ relative error of the PGD-projected function $\tilde{f}_{\pi}^{n}$. The value $q$ denotes the number of terms of the exact function. This function is depicted for the cases $q=\{2,5,11\}$.

must separate the function $f$ with only one term $(n=1)$ independently of the order of the polynomials, i.e. $q$ and $s$. Since the PGD algorithm normalizes the separated functions $F_{\pi, 2}^{1}$ and $F_{\pi, 2}^{1}$, the projection must provide these functions as $F_{\pi, 1}^{1}=P_{q} /\left\|P_{q}\right\|, F_{\pi, 2}^{1}=P_{s} /\left\|P_{s}\right\|$, and the coefficient as $\beta^{1}=\left\|P_{q}\right\|\left\|P_{s}\right\|$. Relative errors below $10^{-15}$ are obtained in these three expressions when solving for (29) using 100 nodes to discretize the 1D domains.

PGD-projection properties can be additionally explored by means of a modification of the function (28), namely $\tilde{f}$. More precisely, only the major order products in the expansion are taken into account, that is

$$
\tilde{f}(x, y)=\sum_{i=0}^{q} x^{i} y^{i}=1+x y+x^{2} y^{2}+\cdots+x^{q} y^{q} .
$$

Note that this function is no longer rank one for $q>1$. Figure 13 shows the relative error of the PGD-projected function $\tilde{f}_{\pi}^{n}$, depicted with respect to the number of projected terms $(n)$. Different values of $q$ in Eq. (30) are studied. The exact function $\tilde{f}$ particularized for the cases $q=\{2,5,11\}$ is also shown.

Results demonstrate that PGD-projection is able to capture the separability of the function $\tilde{f}$ : the number of terms required to reproduce the function $(n)$ is, as maximum, exactly to the number of terms provided $(q)$. Moreover, the PGD-projection leads to compressed expansions for $q>11$, that is, it provides $n<<q$ terms that perfectly capture the function $\tilde{f}$. This is produced because the difference between the last terms of $\tilde{f}$ is 
small, for large values of $q$, and for points in $[0,1]^{2}$.

\section{A.2 Planar waves}

The separability of two planar waves are studied next. The functions to be approximated are

$$
f_{1}(x, k, \theta)=\exp (i k x \cos \theta), \quad f_{2}(x, k, \theta)=\exp (i k x \sin \theta),
$$

with $i=\sqrt{-1}$. The two PGD-projections imposed here separate $x$ from $(k, \theta)$, for instance for the function $f_{1}$ that is

$$
f_{\pi, 1}^{n_{1}}(x, k, \theta)=\pi^{\mathrm{pgd}}\left[f_{1}(x, k, \theta)\right]=\sum_{m=1}^{n_{1}} \beta^{m} F_{\pi, 1}^{m}(x) F_{\pi, 2}^{m}(k, \theta),
$$

and analogously for the function $f_{2}$ and its projection $f_{\pi, 2}^{n_{2}}$. Thus, two separated functions are used and the PGD-projection can be compared with POD using a standard SVD. The spatial coordinate is defined in a unitary domain, $x \in[0,1]$, while $(k, \theta) \in[1,600] \times[\pi, 2 \pi]$. Thus, the number of waves in the spatial domain range from 1 (low-frequency) to 95 (highfrequency). Along each dimension a discretization with $500 \times 100 \times 100$ nodes for $(x, k, \theta)$ is used.

Convergence of the normalized coefficients for both functions $f_{1}$ and $f_{2}$ are shown in Figure 14. For high fidelity purposes (normalized coefficients below $10^{-8}$ ), over 200 terms are necessary when projecting $f_{1}$ while 100 are obtained in the projection of $f_{2}$. The coefficients of the SVD are also depicted in Figure 14 for comparison purposes. Results clearly show that the PGD-projection provide optimal expansions in this case. That is, the greedy procedure is able to find the optimal decomposition that minimizes the distance between $f_{i}$ and $f_{\pi, i}^{n_{i}}, i=\{1,2\}$, in the $\mathcal{L}^{2}$ norm. Note, that every time the PGD-projection is constructed using two separable functions the same coincidence with SVD is observed.

\section{A.3 The butterfly curve}

This last test uses a highly non-separable 6D function based on the family of "butterfly curves", see [25], that is

$$
f(\theta, a, b, c, d, e)=a \exp (\cos \theta)-b \cos (c \theta)+\sin ^{d}(\theta / e),
$$

with $\theta \in[0,2 \pi], a \in[-1,1], b \in[-3,3], c \in[0,4], d \in[0,5]$ and $e \in[1,12]$.

The PGD-projection must seek an approximation in the following form:

$$
f_{\pi}^{n}(\theta, a, b, c, d, e)=\sum_{m=1}^{n} \beta^{m} F_{\pi, 1}^{m}(\theta) F_{\pi, 2}^{m}(a) F_{\pi, 3}^{m}(b) F_{\pi, 4}^{m}(c) F_{\pi, 5}^{m}(d) F_{\pi, 6}^{m}(e) .
$$




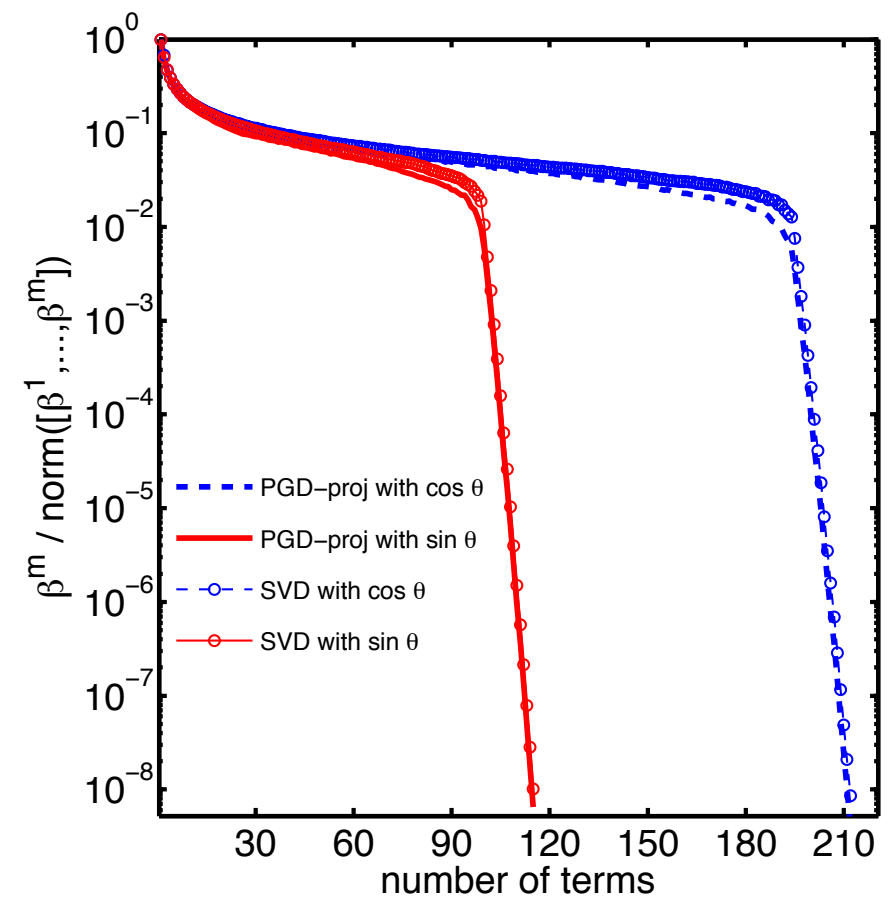

Figure 14: Planar waves: normalized coefficients for the PGD-projection and the SVD vs. number of terms. Exact functions are $f_{1}=\exp (i k x \cos \theta)$ (blue line) and $f_{2}=\exp (i k x \sin \theta)$ (red line).

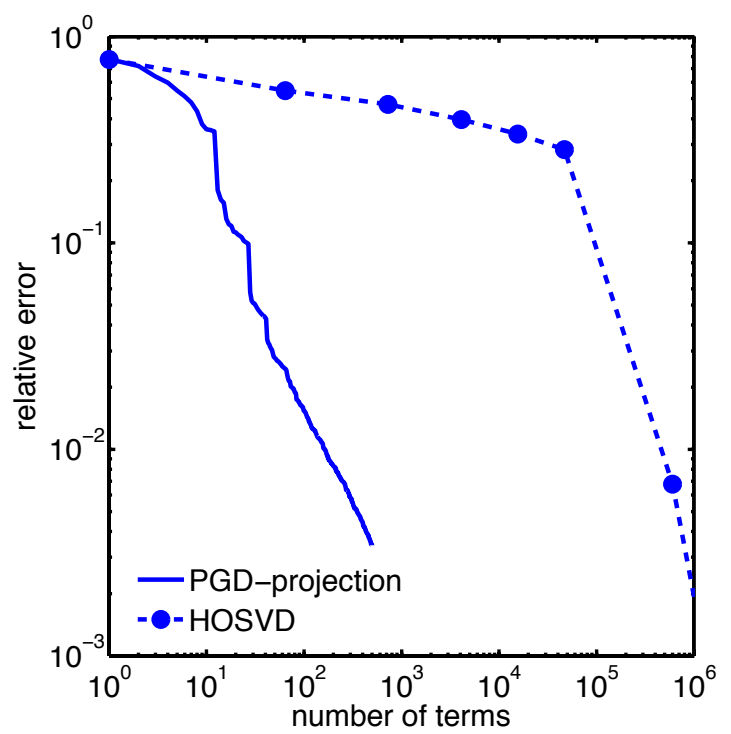

Figure 15: Butterfly curve: relative error of the PGD-projection (solid line) and the $\left(n_{1}, n_{2}, \ldots, n_{6}\right)$ HOSVD (dashed line). Markers on the HOSVD curve represent those cases where $n_{1}=n_{2}=\cdots=n_{6}$. 

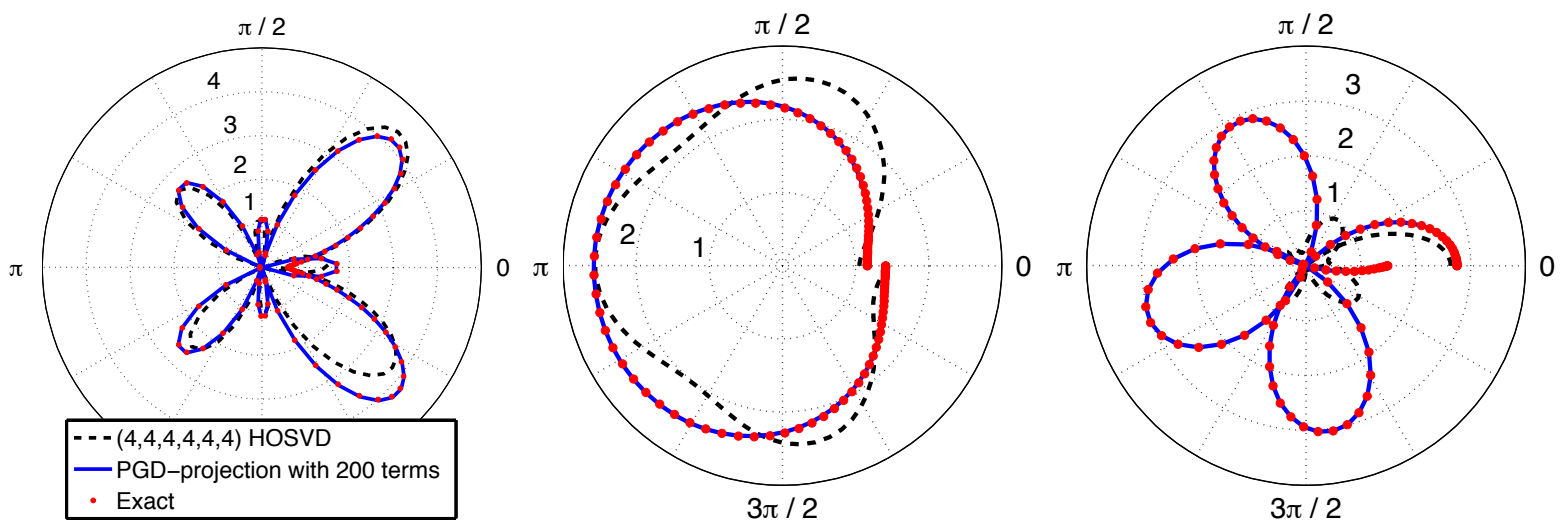

Figure 16: Butterfly curve: polar plots of $f\left(\theta, a^{*}, \ldots, e^{*}\right)$ for fixed values $a^{*}, b^{*}, \ldots, e^{*}$. Approximations given by the PGD-projection (solid line) and the HOSVD (dashed line) are shown. Values of $a^{*}=1, b^{*}=2.1213, c^{*}=4, d^{*}=5, e^{*}=12$ (left), $a^{*}=-0.5736, b^{*}=$ $-2.7189, c^{*}=0, d^{*}=2, e^{*}=11.9791$ (center), and $a^{*}=-0.0872, b^{*}=-2.9886, c^{*}=$ $1.8257, d^{*}=3, e^{*}=7.9235$ (right).

Discretized 6D domain uses 100 nodes along dimension $\theta$, whereas 20 nodes are used for the rest of dimensions but the parameter $d$, that uses 6 equally spaced nodes.

The higher-order approach provided by the standard HOSVD is also computed in this example. Using a similar notation as in Eq. (31), the HOSVD approximation of the function $f$ writes

$$
f_{\text {hosvd }}^{\left(n_{1}, n_{2}, \ldots, n_{6}\right)}(\theta, a, \ldots, e)=\sum_{i=1}^{n_{1}} \sum_{j=1}^{n_{2}} \cdots \sum_{l=1}^{n_{6}} \beta^{i j \ldots l} F_{1}^{i}(\theta) F_{2}^{j}(a) \cdots F_{6}^{l}(e)
$$

The evaluation of $f_{\text {hosvd }}^{\left(n_{1}, n_{2}, \ldots, n_{6}\right)}$ is done by means of tensor decomposition methods, see [36] for details. Note that, for practical purposes, comparison between PGD-projection and HOSVD requires comparing the number of PGD terms $(n)$, and the number of HOSVD terms $\left(n_{1} n_{2} \cdots n_{6}\right)$.

Relative errors of $f_{\pi}^{n}$ and $f_{\text {hosvd }}^{\left(n_{1}, n_{2}, \ldots, n_{6}\right)}$ are depicted in Figure 15. These errors are measured with the $\mathcal{L}^{2}$ norm of the difference between $f$ and both approximations. The often suggested choice $n_{1}=n_{2}=\cdots=n_{6}$ is used for the HOSVD, notwithstanding that other combinations have been also explored with no significant changes in the results. The PGDprojection clearly outperforms the HOSVD, requiring three orders of magnitude less terms to reach the same level of accuracy. The Figure 16 illustrates this conclusion particularizing three polar plots of the curve $f^{*}(\theta)=f\left(\theta, a^{*}, \ldots, e^{*}\right)$ for different fixed values $a^{*}, b^{*}, \ldots, e^{*}$ of the parametric dimensions. Note that abrupt changes in the curve shape between the three cases indicate the highly nonlinear behavior of the exact function $f$. The PGDprojection with 200 terms satisfactory captures the exact values for all the cases, while on the contrary the approximation $f_{\text {hosvd }}^{(4,4, \ldots, 4)}$, that provides 20 times more terms, does not 
exhibit acceptable results specially for the last case. 